\title{
Fibrotic human lung extracellular matrix as a disease-specific substrate for 3D in-vitro models of pulmonary fibrosis
}
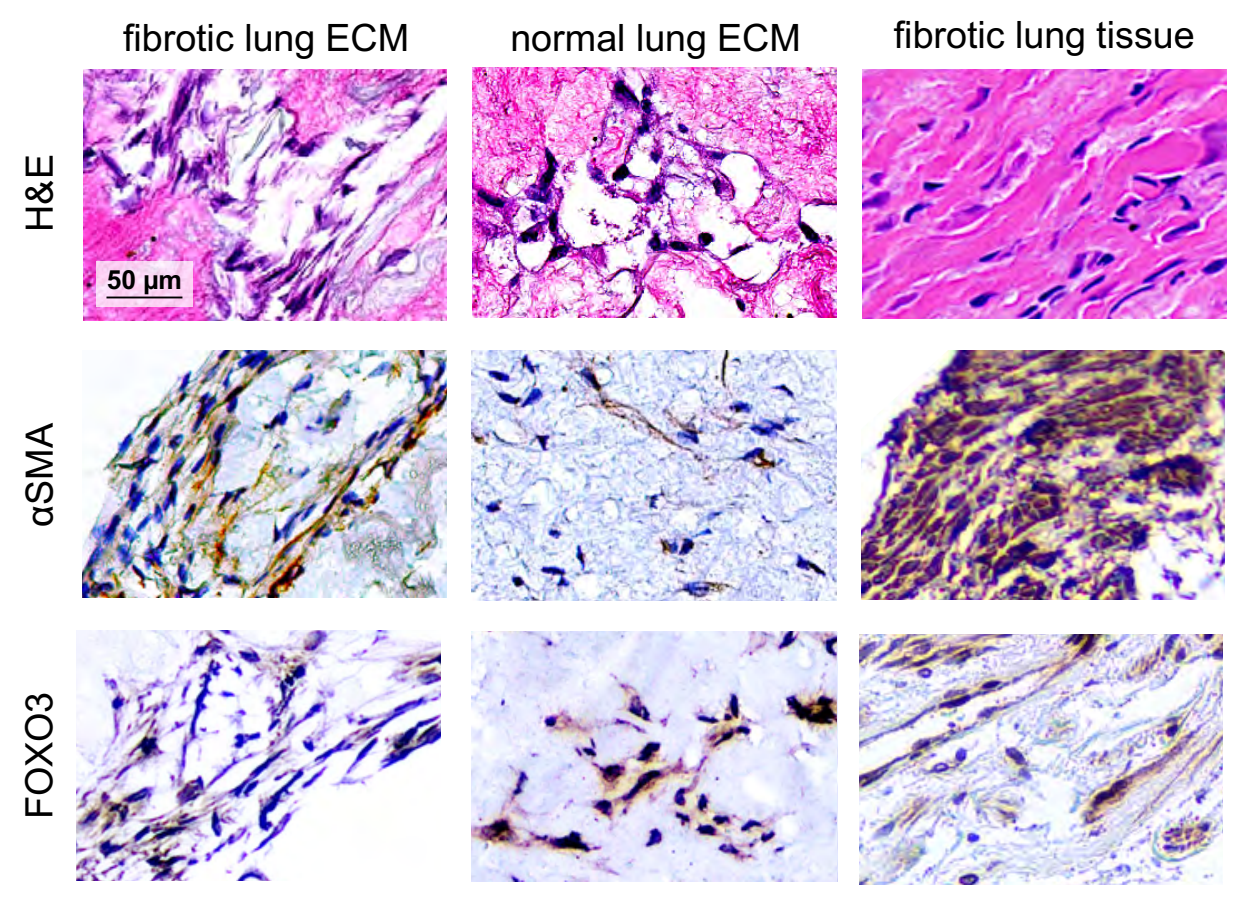

Igal Germanguz ${ }^{\dagger}$, Evelyn Aranda ${ }^{\dagger}$, Jennifer C. Xiong, Natalia Kissel, Alexandra Nichols, Eddie Gadee, John D. O'Neill*

\author{
Xylyx Bio, Inc. \\ 760 Parkside Avenue \\ Brooklyn, New York 11226, USA
}

${ }^{\dagger}$ Authors contributed equally

* Correspondence: John D. O'Neill, Email: johnaxylyxbio.com 
1 Fibrotic human lung extracellular matrix as a disease-specific substrate for 3D in-vitro

2 models of pulmonary fibrosis

4 Igal Germanguz ${ }^{1 \dagger}$, Evelyn Aranda $^{1 \dagger}$, Jennifer C. Xiong ${ }^{1}$, Natalia Kissel $^{1}$, Alexandra Nichols $^{1}$, Eddie

5 Gadee ${ }^{1}$, John D. O’Neill ${ }^{1 *}$

6

$7 \quad{ }^{1}$ Xylyx Bio, Inc., 760 Parkside Avenue, Brooklyn, New York 11226, USA

$8+$ Authors contributed equally

9 * Correspondence: John D. O’Neill, Email: john@xylyxbio.com

\section{ABSTRACT}

12 Idiopathic pulmonary fibrosis (IPF) is an irreversible and uniformly fatal lung disease marked by 13 destruction and scarring of the lung parenchyma and progressive loss of respiratory function. IPF

14 affects nearly 3 million people worldwide, and annual mortality in the US alone exceeds 40,000.

15 Nintedanib and pirfenidone, the only drugs approved for the treatment of IPF, slow progression

16 but do not cure the disease. Consequently, there is a pressing need for effective treatments

17 beside lung transplantation. Unfortunately, predictive models of IPF are not available,

18 underscoring the critical need for physiologically relevant in-vitro substrates that enable

19 quantitative and mechanistic studies of human IPF. Here we report the development and

20 characterization of a human pulmonary fibrosis-specific cell culture substrate comprised of intact

21 fibrotic lung extracellular matrix that recapitulates the human IPF disease environment in vitro.

22 We document the activation and disease-specific phenotype of human lung fibroblasts cultured

23 in the IPF disease-specific substrate, and establish feasibility of testing antifibrotic agents using

24 this substrate. Altogether, our results demonstrate the applicability of this fibrosis-specific

25 substrate for 3D in-vitro models of IPF and cell-based assays in early-stage drug discovery. 
bioRxiv preprint doi: https://doi.org/10.1101/833913; this version posted November 7, 2019. The copyright holder for this preprint (which was not certified by peer review) is the author/funder. All rights reserved. No reuse allowed without permission.

26 Keywords: 3D cell culture, drug testing, extracellular matrix, idiopathic pulmonary fibrosis, in-

27 vitro models, lung disease, lung fibroblasts, scaffolds 


\section{INTRODUCTION}

29 Idiopathic pulmonary fibrosis (IPF) is a chronic interstitial lung disease that primarily affects older

30 adults and is associated with dysregulation of pulmonary fibroblasts, extensive remodeling and

31 deposition of extracellular matrix, and progressive loss of respiratory function. ${ }^{1-3}$ Incidence and

32 prevalence appear to be increasing worldwide with aging populations and improved diagnostics. ${ }^{4}$

33 Every year more than 50,000 new patients are diagnosed with IPF $^{5}$ - an incidence comparable

34 to those of liver, stomach, testicular, or cervical cancers. ${ }^{6}$ After diagnosis, median survival is only

$353-4$ years, and annual mortality exceeds $40,000 .^{7}$ The etiology of IPF remains unknown, but risk

36 factors include smoking, environmental exposures, chronic viral infections, gastroesophageal

37 reflux, lung injury, and genetic predispositions. ${ }^{4,8}$ Nintedanib and pirfenidone, the only drugs

38 approved to treat IPF, attenuate disease progression but do not prevent decline, ${ }^{1,4,9}$ necessitating

39 the development of new drugs that can effectively treat IPF.

41 A major obstacle to developing effective treatments for IPF is the lack of predictive animal and in-

42 vitro models of IPF. Animal models of pulmonary fibrosis are well-established in rodents ${ }^{10-12}$ but

43 present fibrosis that resolves over time rather than the progressive, non-resolving fibrotic process

44 characteristic of IPF in humans. ${ }^{3,13}$ Furthermore, there are no robust or widely adopted in-vitro

45 models of IPF to enable predictive basic and translational studies. ${ }^{14}$ Consequently, an in-vitro

46 model of IPF that emulates human pathophysiology could enable critical new insights into the

47 natural history and pathological mechanisms of IPF, and guide therapeutic development.

49 Current in-vitro models of IPF have limited physiologic relevance because they fail to recapitulate

50 the complex biochemical, structural, and mechanical environment of fibrotic human lungs. In

51 fibrosis, the extracellular matrix $(E C M)$ has different biochemical composition, stores more

52 fibrogenic growth factors, and has altered structure and biomechanics compared to normal

$53 \mathrm{ECM},{ }^{15-17}$ and the direct influence of growth factors ${ }^{18,19}$ and increased matrix stiffness ${ }^{20}$ on 
54 myofibroblast differentiation has been previously demonstrated. Altogether, such matrix

55 alterations induce a profibrotic microenvironment, activate pulmonary fibroblasts, and suggest

56 that IPF progression is correlated with an abnormal ECM microenvironment. ${ }^{21}$ As lung matrix is

57 implicated in both lung function and fibrotic disease progression, IPF models and drug screening

58 platforms not incorporating lung ECM lack defining components of the IPF disease environment.

59 The most common in-vitro IPF drug testing platforms utilize cell culture plates coated with collagen

60 type I and culture media supplemented with high concentrations of transforming growth factor $\beta$

61 (a profibrotic cytokine associated with fibrogenesis), ${ }^{22}$ but no testing platforms that utilize other

62 IPF disease-specific ECM components have been established.

64 An in-vitro cell culture substrate comprised of fibrotic human lung matrix could faithfully recapitulate the composition, structure, and mechanics of the human IPF disease environment. While removal of native cells (decellularization) from human tissues has been demonstrated in a

67 number of tissues including lungs ${ }^{23-26}$, efforts have been primarily focused on the isolation and characterization of ECM from normal, non-diseased tissues. Reproducible, scalable methods for the production of disease-specific ECM biomaterials from diseased human tissues such as fibrotic lungs have not been robustly established. Furthermore, an in-vitro cell culture substrate that

71 recapitulates the complex disease environment of human IPF tissue would be an extremely

72 valuable tool for screening antifibrotic agents in early-stage development.

74 In this study, we investigated the feasibility of developing a cell culture substrate from fibrotic

75 human lung tissue for 3D in-vitro models of human pulmonary fibrosis. Our hypothesis was that

76 normal human lung fibroblasts would display a disease-specific phenotype in vitro in the presence

77 of fibrotic lung extracellular matrix. We implemented a 'physiomimetic approach' to develop

78 disease-specific IPF cell culture substrates (scaffolds) comprised of lung extracellular matrix

79 derived from human IPF tissues (Fig. 1). Our goal was to develop a human fibrotic lung ECM 


\section{Physiomimetic approach for development of disease-specific cell culture substrates}

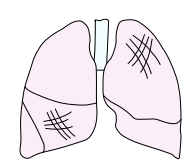

Identify
features of human
disease environment
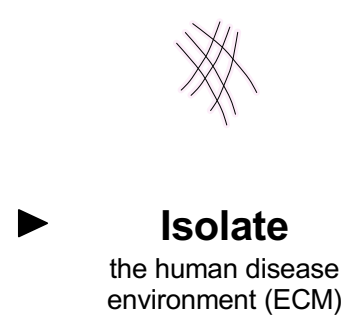

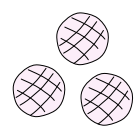

Develop

disease-specific ECM substrate
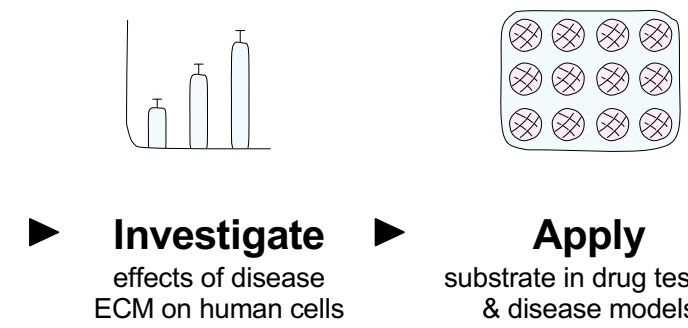

Figure 1 | Overview of physiomimetic approach. Our development of IPF disease-specific cell culture substrates is guided by a physiomimetic approach that aims to identify and isolate the human disease environment, then develop and investigate disease-specific ECM substrates in vitro utilizing disease-relevant human cell types (e.g., pulmonary fibroblasts) whose phenotype can be directly compared against diseased human IPF lung specimens prior to application in IPF disease models and antifibrotic drug testing. 
biomaterial for use as a 3D cell culture substrate for predictive in-vitro models of IPF that could

81 reduce dependence on animal models while enabling physiologically relevant results. Such

82 disease-specific cell culture substrates could radically improve the physiological relevance of in-

83 vitro models of IPF and antifibrotic drug screening platforms, and accelerate development of safe

84 and effective IPF treatments.

\section{MATERIALS \& METHODS}

Procurement of human lung tissues. Acceptance criteria for donors of normal and IPF lungs were established prior to initiation of studies. Normal lung donors had no history, diagnosis, or evidence of: smoking, aspiration pneumonia, asthma, chronic obstructive pulmonary disease, cystic fibrosis, emphysema, interstitial lung disease, or lung cancer. IPF donors required diagnosis of idiopathic pulmonary fibrosis confirmed by a lung transplant pathologist. All IPF donors had end-stage disease and were recipients of lung transplants. Normal human lungs $(n=$ 3) not acceptable for use in transplantation were procured under a protocol approved by the Institutional Review Board at the International Institute for the Advancement of Medicine. Diseased human lungs $(n=3)$ designated as surgical waste were procured under protocols

97 approved by the Institutional Review Boards at Vanderbilt University Medical Center and State 98 University of New York (SUNY) Downstate Medical Center. Lungs were procured in standard 99 fashion, flushed with cold organ preservation solution, transported on ice, and made available 100 without identifiers. In this study, to minimize variability, lung tissues from right middle and right 101 lower lobes were utilized.

103 Characterization of lung donors. Lung donor characteristics were tabulated from deidentified 104 summaries provided by the United Network of Organ Sharing (UNOS) under approved protocols 105 and in compliance with all applicable regulations. 
Sampling of lung tissues. Tissue samples were collected from medial, lateral, and peripheral regions of right middle lobes (2 samples per region), for a total of 6 regional samples per right middle lobe, and 18 regional samples each for IPF and normal lungs.

Preparation of lung matrix scaffolds. Upon receipt, lungs were rinsed with cold sterile saline.

111 Native lung tissue samples were collected for histologic analyses, then lung tissues were stored 112 at $-80^{\circ} \mathrm{C}$. At the time of use, lung tissues were processed under sterile conditions with a 113 proprietary combination of chemicals, enzymes, and surfactants to remove cellular components and isolate normal and fibrotic lung extracellular matrix. Matrix scaffolds (diameter: $7 \mathrm{~mm}$, thickness: $1 \mathrm{~mm}$ ) were prepared under sterile conditions for experimental use. For all assays, three tissue samples or matrix scaffolds were randomly selected from each lung and evaluated in triplicate.

Histologic analyses of lung tissues and scaffolds. Lung tissue samples were fixed in cold

1215 or $10 \mu \mathrm{m}$ thickness. Three sections (medial, lateral, peripheral) from all normal and fibrotic lungs were stained with hematoxylin and eosin, trichrome, Verhoeff-Van Gieson, Alcian blue, and pentachrome, and examined under light microscopy. Representative images were obtained using a fluorescence microscope (FSX100, Olympus).

Histopathologic characterization of lung tissues. All lung sections were subjected to blinded

127 review by a lung transplant pathologist. Slides were randomized, arbitrarily numbered, and 128 delivered without reference to the pathologist, who reviewed and assigned fibrosis scores to all 129 regions in 5 high-power fields according to a standard pulmonary fibrosis scoring rubric ${ }^{27}$ to 130 quantify the extent of architectural disruption and fibrosis (Supplementary Fig. 1C). Fibrosis 131 scores from each high-power field were averaged to obtain an average fibrosis score for each 
132 region of lung. To quantitatively assess the severity and distribution of fibrosis, a grid with unit

133 length $250 \mu \mathrm{m}$ was overlaid onto each high-power field (20x) image, and regions corresponding

134 to various classifications of fibrosis were outlined (Supplementary Fig. 1F). Each region was

135 assigned a calculated relative percent area of the high-power field using the grid. Each fibrosis

136 score was weighted according to percent area, and average fibrosis scores for each high-power

137 field were calculated based on the weighted average of all regional fibrosis scores in each high-

138 power field. Fibrosis scores were then averaged across 5 high-power fields per region, with four

139 regions evaluated per lobe. Only regions of IPF lungs with confirmed fibrosis score $\geq 2$ were

140 investigated in this study (Supplementary Fig. 1D,E,G).

Biochemical characterization of lung tissues and scaffolds. To quantify collagen in lung tissues and scaffolds, samples were weighed, homogenized, and digested with pepsin $(0.1 \mathrm{mg}$

$144 \mathrm{~mL}^{-1}$ ) in $0.5 \mathrm{M}$ acetic acid for 12 hours at $4^{\circ} \mathrm{C}$, and subjected to a collagen quantification assay 145 (Sircol, Biocolor) according to the manufacturer's instructions. To quantify sulfated glycosaminoglycans in lung tissues and scaffolds, samples were weighed, homogenized, and 147 digested with papain $\left(1 \mu \mathrm{g} \mathrm{mL}^{-1}\right)$ for 12 hours at $60^{\circ} \mathrm{C}$, and subjected to the dimethylene blue dye 148 assay, wherein absorbance was measured at $595 \mathrm{~nm}$. To quantify elastin in lung tissues and 149 scaffolds, samples were weighed and homogenized, and soluble a-elastin was extracted via 150 three extractions with hot $0.25 \mathrm{M}$ oxalic acid. Samples were then subjected to an elastin 151 quantification assay (Fastin, Biocolor) according to the manufacturer's instructions. To quantify 152 residual DNA in matrix scaffolds, samples were subjected to a quantitative DNA assay (Quant-iT 153 PicoGreen, Invitrogen) according to the manufacturer's instructions.

155 Immunohistochemical staining. Following de-paraffinization, sections of lung tissues and 156 scaffolds were subjected to boiling citrate buffer $(\mathrm{pH} 6.0)$ for antigen retrieval, and blocked with $1575 \%$ normal goat serum in phosphate-buffered saline for 1 hour at room temperature. Next, 
antibodies were diluted as necessary, applied, and incubated for 12 hours at $4^{\circ} \mathrm{C}$ or 4 hours at room temperature. Sections were mounted (VectaMount Permanent Mounting Medium, Vector Laboratories), and coverslips were applied. Images were obtained using a light microscope

161 (Eclipse Ts2, Nikon). Immunohistochemical stains were performed for alpha smooth muscle actin 162 (Cell Signaling Technology, 19245), fibrillin 2 (Sigma Life Science, HPA012853), Ki67 163 (ThermoFisher Scientific, PA1-38032), laminin y1 (Abcam, ab233389), matrix gla protein (LS Bio, 164 LS-B14824), and periostin (Abcam, ab14041). A list of antibodies with dilutions used is provided 165 in Supplementary Table 1.

Quantification of immunohistochemical staining by image analysis. Images of immunohistochemical stains were captured using a slide scanner (P250 High Capacity Slide Scanner, 3D Histech). To quantify immunohistochemical staining, images were analyzed using an image analysis software module (DensitoQuant, Quant Center, 3D Histech), and the number of positive 171 and negative pixels were quantified and analyzed.

Mass spectrometry of IPF and normal lung matrisomes. Detailed methods are available in the

174 Supplementary Information.

176 Quantification of growth factors. To quantify growth factors in native lung tissues and matrix 177 scaffolds, a multiplex growth factor array (Quantibody Human Growth Factor Array Q1; Ray 178 Biotech) was performed and analyzed by Q-Analyzer software. To quantify growth factors 179 secreted by human fibroblasts in vitro, enzyme-linked immunosorbent assays (ELISA) were 180 performed for bFGF (R\&D Systems, DFB50) and TGF $\beta$ (R\&D Systems, DB100B). All samples 181 were analyzed in triplicate. 
Scanning electron microscopy. Lung matrix samples were collected, fixed in formalin for 24 hours, rinsed in $70 \%$ ethanol, frozen, lyophilized, and imaged using an electron microscope

184 (GeminiSEM 300, Zeiss) with accelerating voltage $2.5 \mathrm{kV}$.

Transmission electron microscopy. Lung matrix samples were fixed with $2.5 \%$ glutaraldehyde, $4 \%$ paraformaldehyde, and $0.02 \%$ picric acid in $0.1 \mathrm{M} \mathrm{Na-cacodylate} \mathrm{buffer} \mathrm{(} \mathrm{pH} 7.2)$. Samples were then post-fixed with $1 \%$ OsO4 in Sorenson's buffer for 1 hour, dehydrated, and embedded in Lx-112 (Ladd Research Industries). Sections (thickness: $60 \mathrm{~nm}$ ) were prepared using a PT-XL ultramicrotome, stained with uranyl acetate and lead citrate, and examined with an electron microscope (JEM-1200 EXII; JEOL). Images were captured with a digital camera (ORCA-HR; Hamamatsu Photonics) and recorded with imaging software (Image Capture Engine, AMT).

Mechanical testing of IPF and normal lung scaffolds. Uniaxial tensile mechanical testing was conducted with a $10 \mathrm{~N}$ load cell (Model 5848, Instron), as previously described. ${ }^{23}$ Lung tissues and matrix from transverse sections of the right middle lobe were randomly selected and dissected into $3 \mathrm{~cm}$ by $1 \mathrm{~cm}$ samples. A consistent orientation from right middle lobe was maintained to minimize effects of lung anisotropy on mechanical testing data. Samples were secured and mounted, and a pre-load of $0.003 \mathrm{~N}$ was applied. All samples were tested at the same grip-to-grip distance for consistency. Samples were kept hydrated throughout all mechanical testing with phosphate-buffered saline at room temperature. A $20 \%$ uniaxial strain 202 was applied at a strain rate of $1 \% \mathrm{~s}^{-1}$, and at frequencies of $0.25,0.50$, or $0.75 \mathrm{~Hz}$.

204 Cell culture. Human lung fibroblasts (ATCC) were cultured in Dulbecco's Modified Eagle Medium 205 (DMEM) supplemented with 10\% fetal bovine serum and 1\% penicillin/streptomycin under 206 standard culture conditions with $5 \% \mathrm{CO}_{2}$ at $37^{\circ} \mathrm{C}$. 
Gene expression analysis. Total RNA was extracted (RNeasy Micro Kit, QIAGEN), and cDNA synthesis was performed using random primers (iScript Select cDNA Synthesis Kit, Bio-Rad). Quantitative real-time polymerase chain reaction (qPCR) was performed in triplicate using master mix (Brilliant III Ultra-Fast SYBR Green QPCR Master Mix, Agilent Technologies) and a real-time PCR system (AriaMax Real PCR System, Agilent Technologies). A list of primers is provided in

\section{Supplementary Table 2.}

Drug testing. Normal human fibroblasts were cultured in vitro for 24 hours, then exposed to antifibrotic agent PF3644022 hydrate (PZ-0188, Sigma-Aldrich) at a concentration of $1 \mu \mathrm{M}$ for 72 hours. Metabolic activity was measured using Alamar Blue reagent (DAL1025, ThermoFisher Scientific) according to the manufacturer's instructions. The reagent was added to cells in culture at 24, 48 and 72 hours, and incubated for 4 hours before readout. Absorbance was measured at $570 \mathrm{~nm}$, with reference wavelength at $600 \mathrm{~nm}$.

Statistical analyses. One-way ANOVA and Student's $t$-tests were performed using statistical analysis software (Prism 8, GraphPad), and $p<0.05$ was considered significant.

\section{RESULTS}

Assessment of IPF and normal lungs. Donor characteristics of IPF and normal lung tissues were analyzed to confirm that there were no significant differences in age, height, weight, body mass index, or smoking history (Supplementary Fig. 1A,B; Supplementary Table 3). An established numerical rubric ${ }^{27}$ was used to assess the extent of histomorphologic disruption and

230 fibrosis. Tissue sampling and histopathologic analyses are described in detail in Supplementary 231 Information. 
232 Preparation of lung matrix scaffolds. Native lung tissues were treated with a proprietary

233 combination of chemicals, enzymes, and surfactants to remove cellular and nuclear components,

234 which was confirmed by hematoxylin and eosin staining (Fig. 2A) and quantitative DNA assay

235 (Supplementary Fig. 2A). Matrix scaffolds from all human lungs were confirmed negative for

236 mycoplasma, bacteria, and fungi (Supplementary Fig. 2B), and deemed suitable for use in cell-

237 based studies.

239 IPF matrix scaffolds recapitulate disease-specific histologic features. For histologic

240 evaluations of IPF, representative fields corresponding to fibrosis score 3 (severe fibrosis) were

241 selected. To visualize distributions of ECM structural components in IPF and normal lungs,

242 histologic staining was performed on native (untreated) tissues and matrix scaffolds. H\&E staining

243 of native IPF tissues revealed severe distortion of lung structure and large areas of fibrous

244 obliteration with minimal remaining airspace (Fig. 2A). By contrast, H\&E staining of native normal

245 lung tissues displayed abundant airspaces defined by thin alveolar septa and stereotypical

246 alveolar saccular architecture. Matrix scaffolds from analogous regions of IPF and normal lungs

247 had no discernible nuclei and displayed drastic differences in scaffold architecture consistent with

248 fibrotic and normal native lung tissues, respectively. Trichrome staining showed dramatic

249 deposition of collagens (blue) throughout regions of severe fibrosis (Fig. 2B). In IPF tissues and

250 scaffolds, collagen fibers were observed in densely aligned bundles and in loosely disorganized

251 networks; whereas in normal lung tissues and scaffolds, collagen was organized along alveolar

252 septa and within the interstitium. Verhoeff-Van Gieson (VVG) elastic staining showed a notable

253 loss of elastic fibers (black) in regions of IPF tissues and scaffolds with severe fibrosis, whereas

254 in normal lung tissues and scaffolds elastic fibers were dispersed homogenously throughout the 255 respiratory zone (Fig. 2C). 


\section{native tissues}

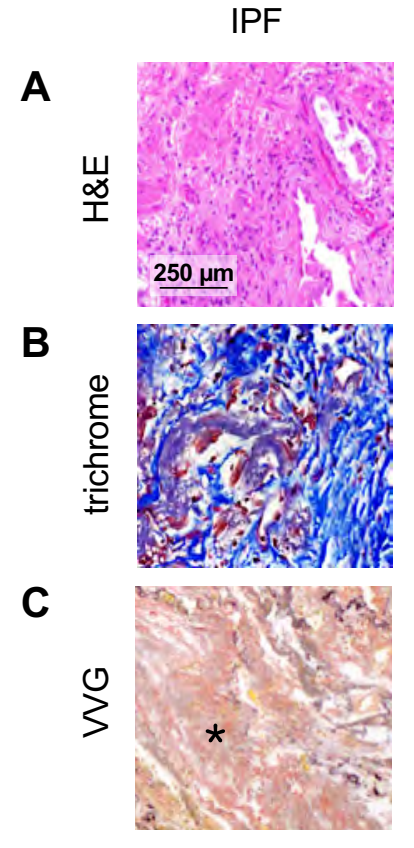

normal
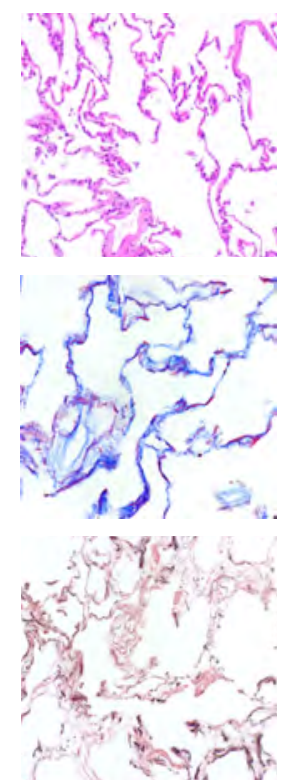

D

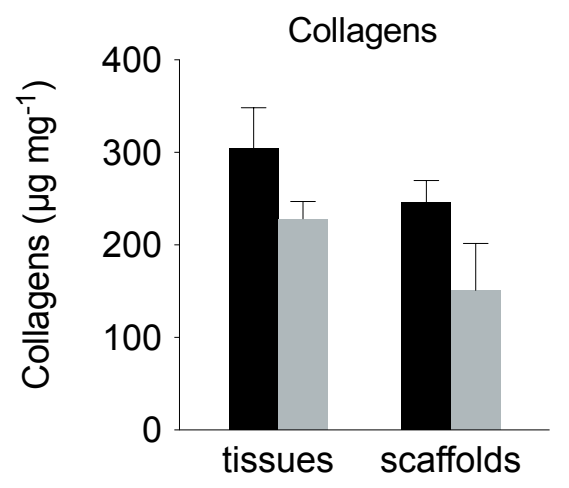

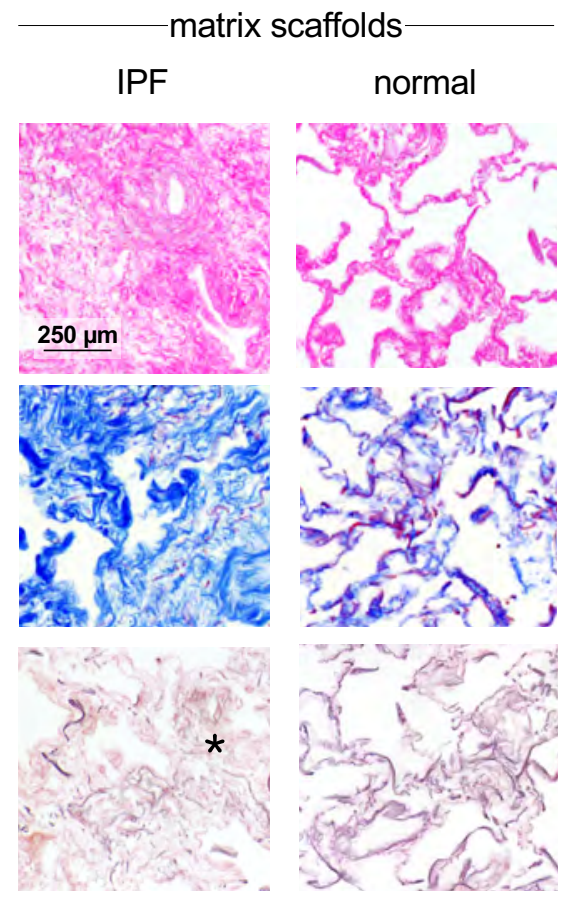

E

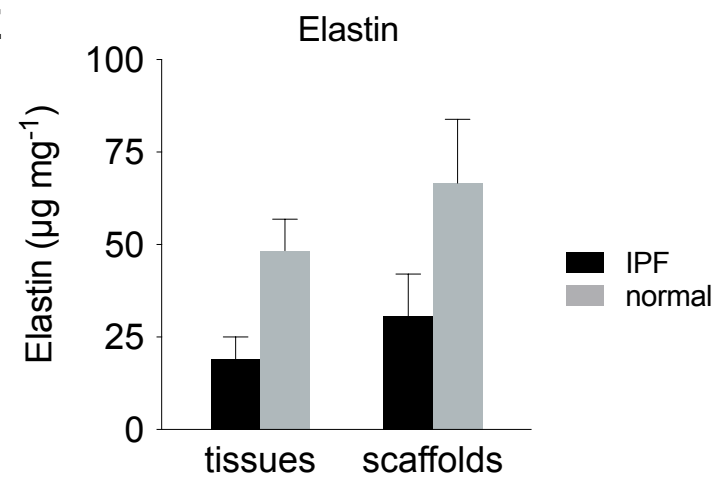

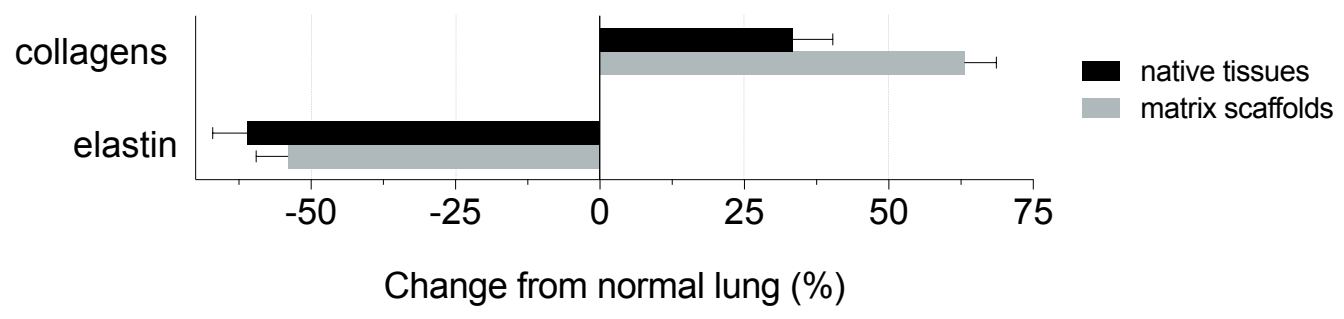

Figure 2 | Histologic \& biochemical characterization of ECM structural components in IPF and normal lung tissues and matrix scaffolds. Representative micrographs of histologic stains: (A) hematoxylin and eosin (H\&E), (B) trichrome (collagens, blue), and (C) Verhoeff-Van Gieson (VVG, elastic fibers, black) demonstrating differences in histomorphology of IPF and normal lung tissues and scaffolds. Star indicates representative region with severe fibrosis and loss of elastic fibers. Quantification of structural ECM components (D) collagens and (E) elastin by biochemical assays. (F) Changes from normal lung in structural ECM components in IPF. H\&E: hematoxylin and eosin, VVG: Verhoeff-Van Gieson. 
IPF matrix scaffolds contain disease-specific biochemical composition. Soluble collagens

were quantified in native tissues and matrix scaffolds, and increases in collagens were measured relative to normal in IPF native tissues $(33.3 \pm 19.2 \%)$ and matrix scaffolds $(63.2 \pm 15.6 \%$, Fig. 2D). Consistent with the loss of elastic fibers observed in VVG elastic staining, quantification of elastin confirmed reduction in IPF native tissues $(60.6 \pm 12.3 \%)$ and matrix scaffolds $(54.1 \pm 17.2$ \%) relative to normal (Fig. 2E). Altogether, the structural ECM components in IPF demonstrated clear trends relative to normal in both native tissues and matrix scaffolds: increased collagens (33 - 63\%) and decreased elastin (54-61\%; Fig. 2F). Alcian blue and pentachrome staining were performed to assess the extent and distribution of proteoglycans in IPF tissues, which was significantly higher in areas of moderate and severe fibrosis (scores $\geq 2$ ) than in areas of mild fibrosis (scores < 2) and normal lung tissues (Fig. 3A,B). Quantification of sulfated glycosaminoglycans (GAG) revealed that GAG components in IPF native tissues and scaffolds was $232.5-300.5 \%$ higher than in normal lungs (Fig. 3C-E), consistent with overexpression of sulfated glycosaminoglycans previously observed in fibrotic foci ${ }^{28}$. Immunohistochemical staining

271 of IPF tissues for multiple ECM glycoproteins revealed dramatic differences from normal lung

272 tissues in fibrillin 2, laminin $\mathrm{Y1}$, matrix GLA protein (MGP), and periostin (Fig. 3F-I). Areas with

273 severe fibrosis (fibrosis score: 3 ) were characterized by pervasive overexpression of fibrillin 2,

274 MGP, and periostin, and loss of laminin y1. Notably, changes from normal lung were consistent

275 in native tissues and matrix scaffolds for all glycoproteins that were investigated (Fig. 3J).

277 Mass spectrometry was performed on IPF and normal lung matrix scaffolds to assess the IPF

278 matrisome (Table 1), and revealed changes from normal lung consistent with histopathologic 279 observations and biochemical assays. Multiple collagen types increased above 150\%, including 280 collagen types I, II, V, VI, VIII, XVI. Notably, in IPF lungs collagen types IV and XXI - the primary 281 collagens comprising the alveolar basement membrane - decreased between $33-73 \%$, 282 consistent with the loss of basement membrane and alveolar structure associated with the 
IPF tissue

A

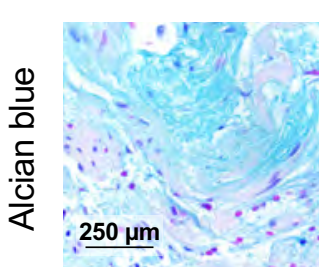

B

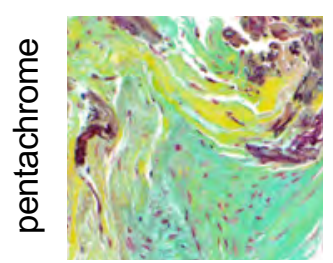

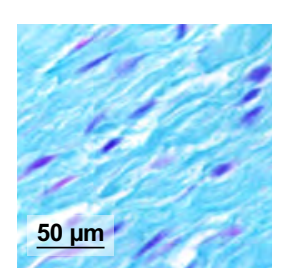

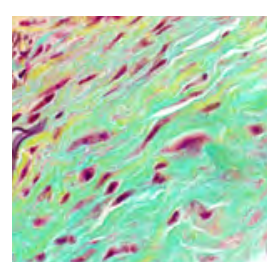

normal tissue

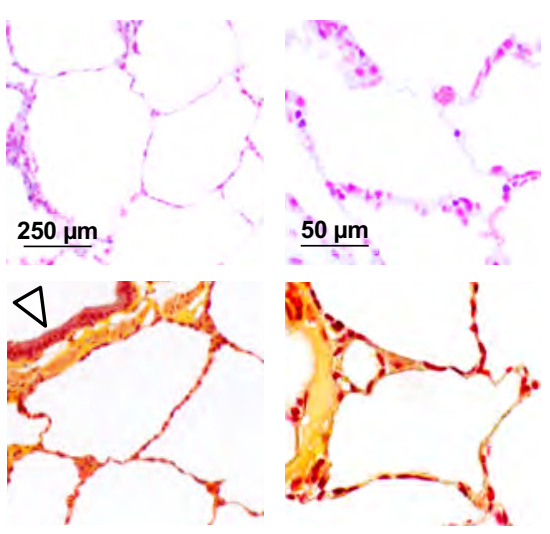

E

Glycosaminoglycan ECM components in IPF

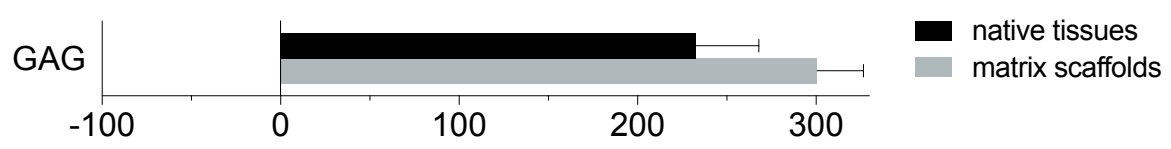

Change from normal lung $(\%)$ native tissues

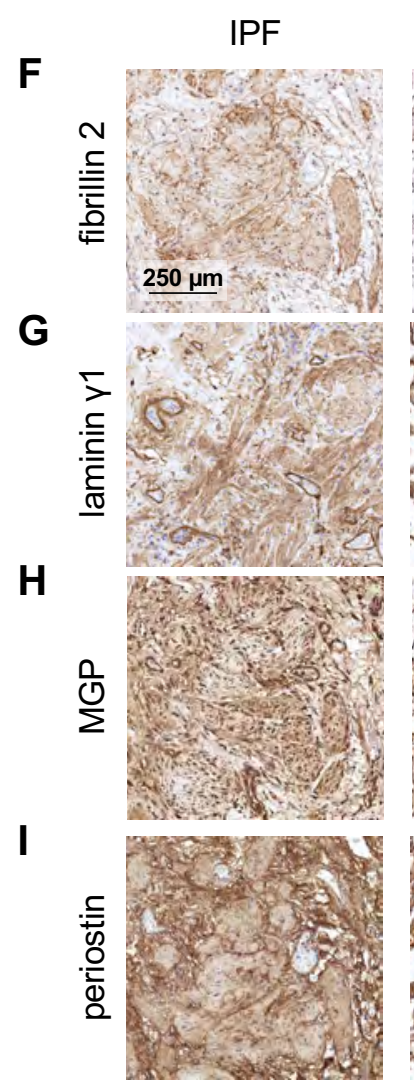

matrix scaffolds

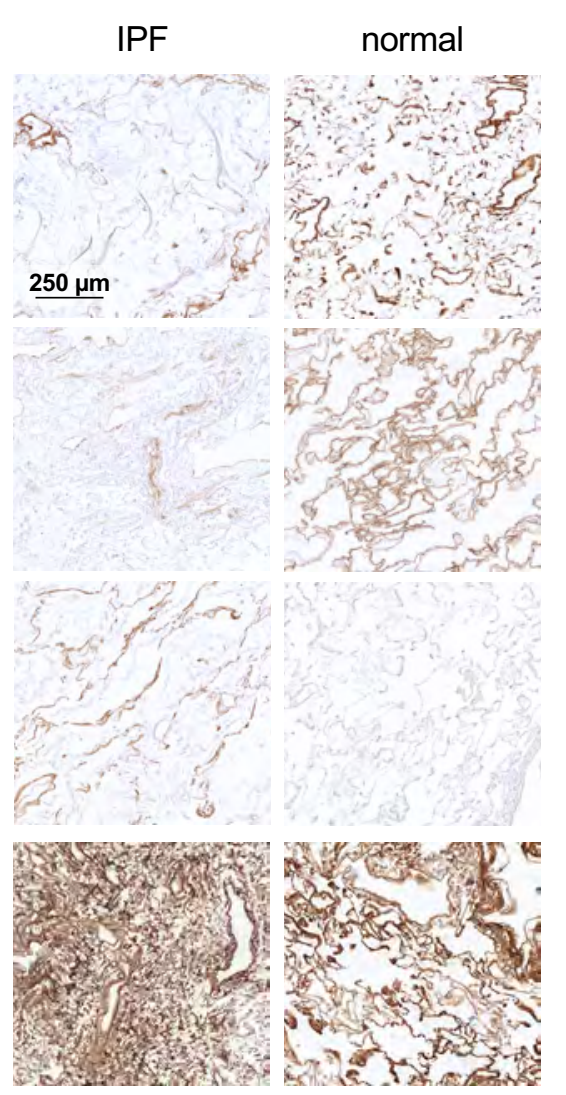

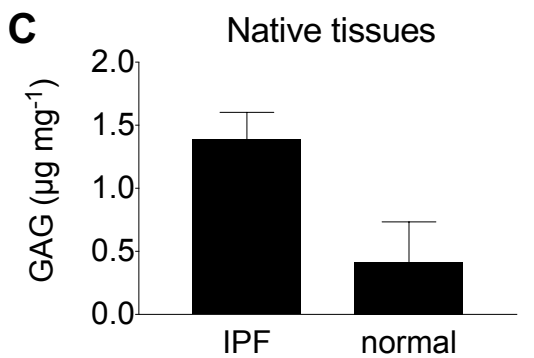

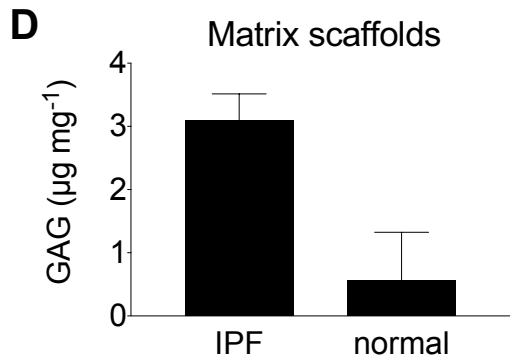

Glycoprotein ECM components in IPF

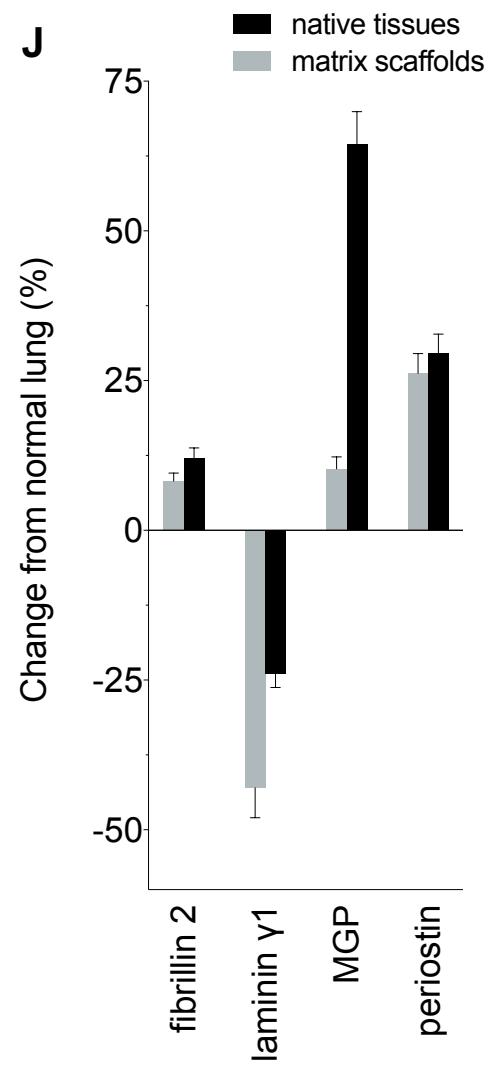

Figure 3 | Characterization of proteoglycans and glycoproteins in IPF lung tissues and matrix scaffolds. Representative micrographs of histologic stains: (A) Alcian blue (proteoglycans, blue) and (B) pentachrome (acidic polysaccharides, green) demonstrating differences in proteoglycans between IPF and normal lung tissues. Arrow indicates normal airway epithelium. Quantification of sulfated glycosaminoglycan ECM components in (C) native tissues and (D) matrix scaffolds. (E) Changes from normal lung in glycosaminoglycan ECM components in IPF. Immunohistochemical staining of glycoprotein ECM components in IPF: (F) fibrillin 2, (G) laminin Y1, (H) matrix gla protein (MGP), (I) periostin. (J) Quantification of glycoproteins by image analysis of immunohistochemical staining using DensitoQuant software. 


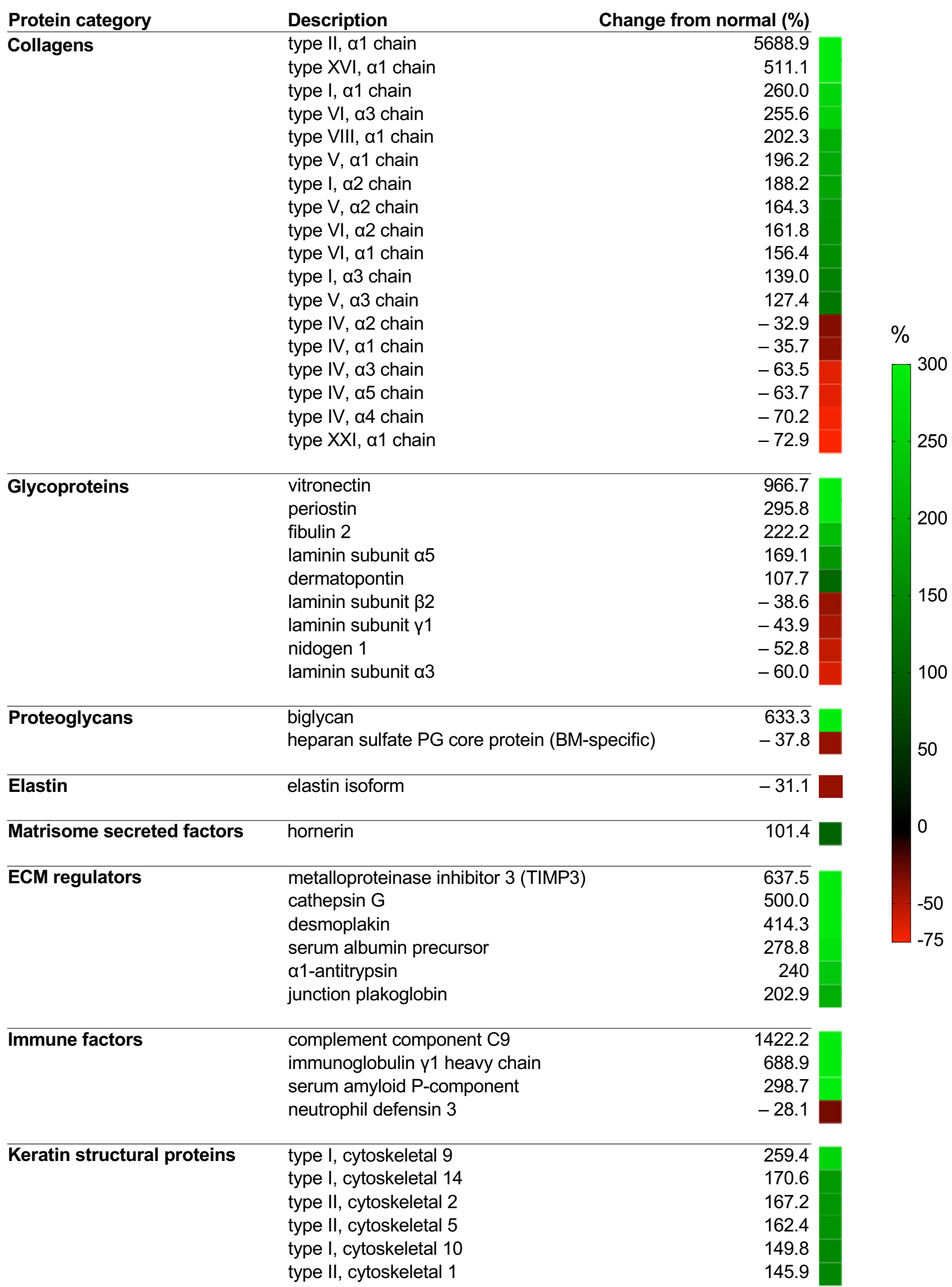

Table 1 | Mass spectrometry summary analysis of IPF lung matrisome. Changes from normal in the abundance of IPF lung matrisome components. PG: proteoglycan, BM: basement membrane, 
283 progression of pulmonary fibrosis. ${ }^{29}$ The glycoprotein vitronectin was elevated $967 \%$, and

284 glycoproteins fibulin 2 and periostin were both elevated above $200 \%$. Laminin subunits $\alpha 3, \beta 2$,

$285 \mathrm{Y} 1$, and nidogen 1, which are associated with the basement membrane, were all decreased in IPF

286 lungs. Biglycan was increased by $633 \%$, however basement membrane-specific heparan sulfate

287 proteoglycan core protein was decreased by $38 \%$. Elastin isoforms were also decreased by $31 \%$,

288 consistent with quantitative biochemical analyses. Interestingly, in IPF lungs several regulators of

289 the extracellular matrix were also increased more than $200 \%$ above normal, including

290 metalloproteinase inhibitor 3 (TIMP3), cathepsin G, desmoplakin, and a1-antitrypsin.

292 To assess changes in endogenous growth factors, a multiplex growth factor array was performed.

293 Two growth factors were detected only in IPF native tissues and not in normal lung native tissues:

294 transforming growth factor beta 3 (TGF- $\beta 3$ ) and heparin-binding EGF-like growth factor (HB-EGF;

295 Supplementary Table 4). In IPF native tissues, insulin-like growth factor binding protein 1

296 (IGFBP-1) was 160-fold above normal, and both basic fibroblast growth factor (bFGF) and

297 endocrine gland-derived vascular endothelial growth factor (EG-VEGF) were approximately 20-

298 fold above normal. Brain-derived neurotrophic factor (BDNF) and growth differentiation factor 15

299 (GDF-15, a prognostic factor for $\mathrm{IPF}^{30}$ ) were elevated 3-fold to 5-fold, but osteoprotegerin (OPG)

300 was reduced by more than half. Five growth factors were detected in IPF matrix scaffolds (Table

301 2), including IGFBP-6, whose family of carrier proteins were shown to induce production of

302 collagen type I and fibronectin in normal primary lung fibroblasts ${ }^{31,32}$. Neurotrophin-4 (NT-4),

303 which is elevated in explanted IPF lungs and shown to drive proliferation of primary human lung

304 fibroblasts through TrkB-dependent and protein kinase B-dependent pathways ${ }^{33}$, was also 305 detected in IPF matrix scaffolds.

307 IPF matrix scaffolds have disease-specific structural and mechanical properties. The gross

308 appearance of IPF matrix scaffolds was dramatically different from the appearance of normal lung 


\begin{tabular}{|c|c|c|c|c|}
\hline \multirow[b]{2}{*}{ Growth factor } & \multirow[b]{2}{*}{ Description } & \multicolumn{2}{|c|}{ Concentration (pg mL $\left.{ }^{-1}\right)$} & \multirow{2}{*}{$\begin{array}{l}\text { Fold change } \\
\text { from normal }\end{array}$} \\
\hline & & Normal scaffold & IPF scaffold & \\
\hline GDF-15 & Growth differentiation factor 15 & 0.8 & 14.5 & $+18.1 \Delta$ \\
\hline BDNF & Brain-derived neurotrophic factor & 6.3 & 48.7 & $+7.7 \Delta$ \\
\hline IGFBP-6 & Insulin-like growth factor binding protein 6 & 14.1 & 71.8 & $+5.1 \Delta$ \\
\hline HGF & Hepatocyte growth factor & 23.8 & 91.0 & $+3.8 \Delta$ \\
\hline EG-VEGF & Endocrine gland-derived vascular endothelial growth factor & 0.6 & 1.5 & $+2.5 \Delta$ \\
\hline bFGF & Basic fibroblast growth factor & 22.6 & 54.8 & $+2.4 \Delta$ \\
\hline HB-EGF & Heparin-binding EGF-like growth factor & 1.4 & 2.5 & $+1.8 \Delta$ \\
\hline TGF- $\beta 3$ & Transforming growth factor $\beta 3$ & 2.8 & 4.0 & $+1.4 \Delta$ \\
\hline VEGF & Vascular endothelial growth factor & 4.6 & 3.0 & $-0.3 \nabla$ \\
\hline EGF R & Epidermal growth factor receptor & ND & ND & - \\
\hline
\end{tabular}

Table 2 | Quantification of growth factors in IPF and normal lung matrix scaffolds. Growth factor concentrations were measured by multiplex growth factor array. Green arrow $(\boldsymbol{\Delta})$ indicates positive fold change (increase) from normal in concentration of growth factors. Red arrow $(\nabla)$ indicates negative fold change (decrease) from normal in concentration of growth factors. ND: not detected. 
matrix scaffolds. Normal lung matrix scaffolds appeared translucent, with visible bronchial and vascular conduits and saccular structures throughout the parenchyma (Fig. 4A). By contrast, IPF matrix scaffolds had pervasive dense fibroconnective structures, with abnormal disorganized architecture, honeycombing, and no apparent airways or vessels. Scanning electron microscopy revealed dramatic disruption of normal alveolar architecture in IPF scaffolds (Fig. 4B).

314 Topography of collagen fibers in IPF scaffolds was visualized by inverted color micrographs of 315 trichrome staining, which showed dense fibrous bundles in IPF scaffolds and stereotypical porous 316 (alveolar-like) networks in normal lung scaffolds (Fig. 4C). Transmission electron microscopy 317 showed dense fibrous bands $(F)$ of extracellular matrix in IPF matrix scaffolds with minimal evidence of normal basement membrane, whereas normal lung matrix scaffolds had abundant

319 airspaces (A), delicate basement membrane (arrow), and alveolar capillaries (C; Fig. 4D). Uniaxial mechanical testing of IPF and normal tissues and scaffolds indicated that IPF tissues and scaffolds were approximately $20 \times$ stiffer at $5 \%$ strain and approximately $5 \times$ stiffer at $20 \%$ strain compared to normal tissues and scaffolds (Fig. 4E). Importantly, mechanical testing also confirmed that the processing of native tissues to obtain matrix scaffolds did not alter the mechanical properties of matrix scaffolds from native tissues, as differences in elastic modulus between native tissues and matrix scaffolds were not significant (Fig. 4F,G). H\&E staining showed that the phenotype of normal human lung fibroblasts varied between cells 330 cultured in IPF and normal lung matrix scaffolds (Fig. 5A). Fibroblasts in IPF matrix scaffolds 331 showed higher expression of alpha smooth muscle actin than fibroblasts in normal lung matrix 332 scaffolds. Morphologic similarities between fibroblasts cultured in IPF scaffolds and IPF native 333 tissue were observed (Fig. 5B). In contrast, immunostaining of FOXO3, a transcription factor 334 whose downregulation is linked to fibrogenesis ${ }^{34}$, showed lower expression in human lung 
IPF scaffolds

A

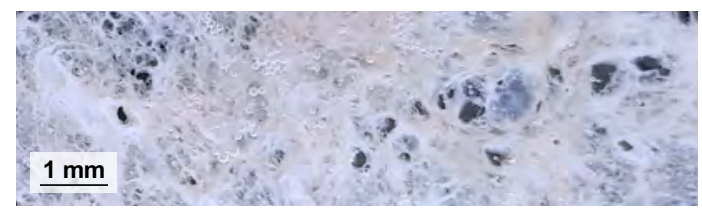

B

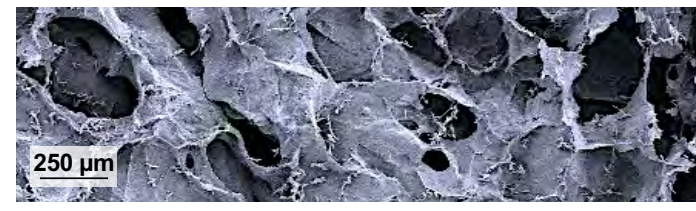

C

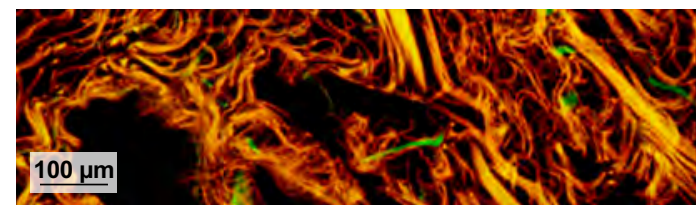

D

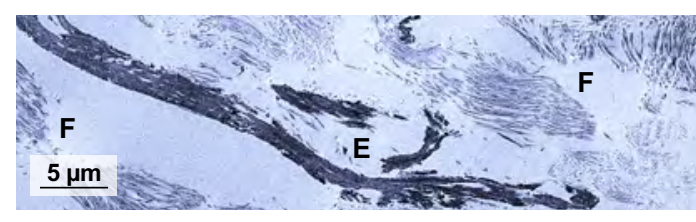

E

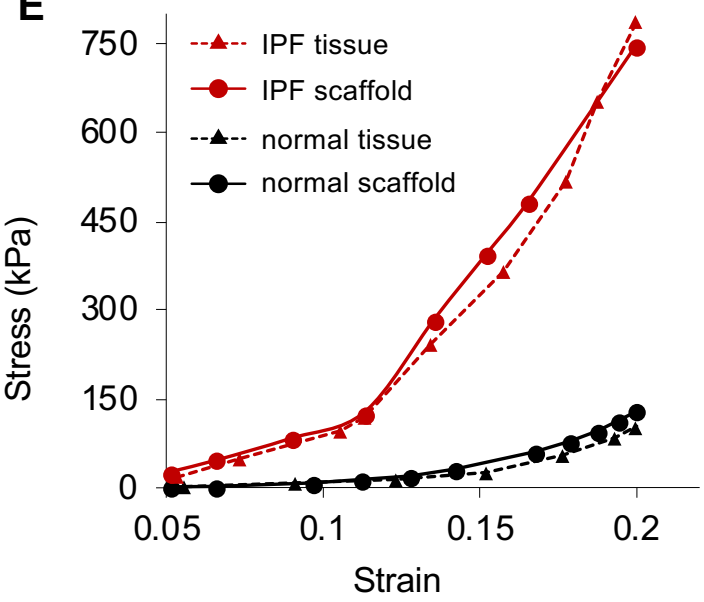

normal scaffolds
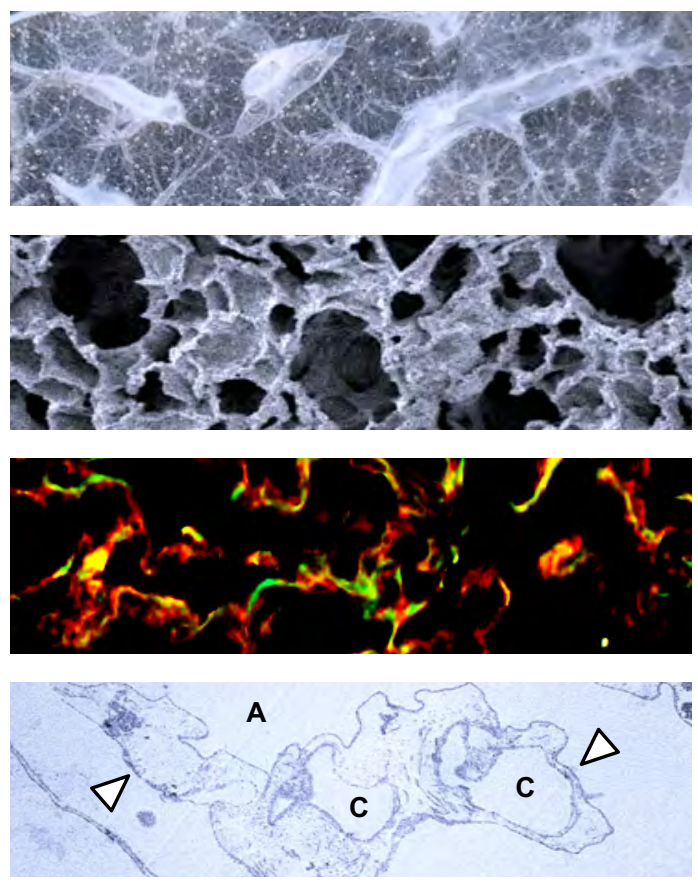

$\mathbf{F}$

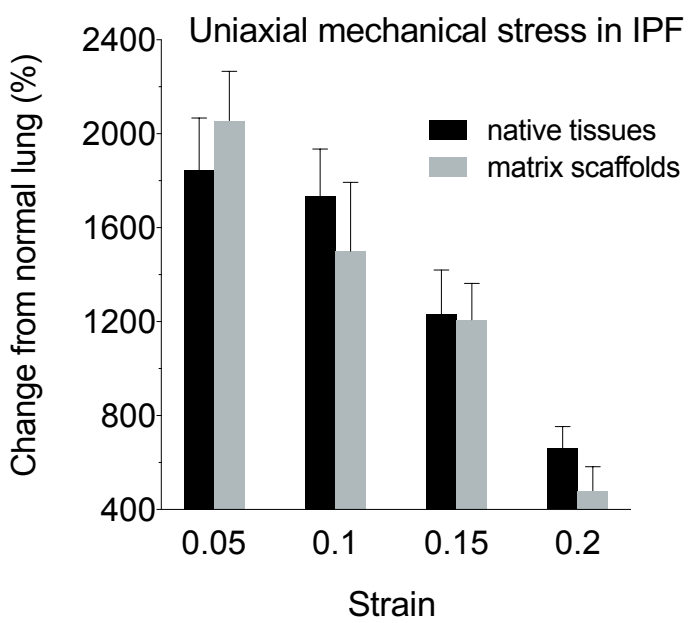

G

\begin{tabular}{rr} 
IPF tissue & IPF scaffold \\
\hline $10,070.3 \pm 3347.3$ & $7218.5 \pm 3119.5$ \\
0.989 & 0.988 \\
\hline 0.24 &
\end{tabular}

\begin{tabular}{rr} 
normal tissue & normal scaffold \\
\hline $1574.9 \pm 326.3$ & $2325.4 \pm 455.1$ \\
0.977 & 0.998 \\
\hline & 0.11
\end{tabular}

p-value

0.11

Figure 4 | Structural, topographical, and mechanical characterizations of IPF lung scaffolds. Representative images of IPF and normal lung scaffolds: (A) gross photography, (B) scanning electron microscopy, (C) light microscopy (inverted color micrograph) of trichome staining demonstrating topography of ECM fibers in IPF scaffolds, (D) transmission electron microscopy. A: airspace, C: alveolar capillary, E: elastin bundle fragments, F: fibroconnective collagenous matrix, arrow: basement membrane. (E) Representative uniaxial stress-strain curves of IPF and normal lung tissues and matrix scaffolds. (F) Change in uniaxial mechanical stress from normal lung tissues and matrix scaffolds. (G) Tangent modulus values. Statistical analyses between tissues and scaffolds were performed using Student's $t$-test, with significance when $p<0.05$. All values represent mean \pm standard deviation. 
335 fibroblasts cultured on IPF matrix scaffolds compared to fibroblasts cultured on normal lung matrix

336 scaffolds (Fig. 5C). Consistent with alpha smooth muscle immunohistochemical staining, gene

337 expression analysis showed significant upregulation of ACTA2 (alpha smooth muscle actin).

338 Additional upregulated fibrosis-specific markers of fibroblast activation included COL1A1

339 (collagen type I, subunit a1), MMP2, PDGFC, PTEN, and PRRX1 (Fig. 5D). Activation of

340 fibroblasts in vitro was also assessed by quantification of secreted basic fibroblast growth factor

341 (bFGF) and transforming growth factor beta (TGF $\beta$ ), with normal human lung fibroblasts cultured

342 on tissue culture plastic as a standard control. Interestingly, secretion of bFGF and TGF $\beta$ were

343 both highest with fibroblasts cultured in IPF matrix scaffolds (Fig. 5E,F). Notably, secreted TGF $\beta$

344 was significantly higher in IPF matrix scaffolds compared to normal lung matrix scaffolds,

345 suggesting that substrate stiffness may have influenced secretion of TGF $\beta$.

IPF matrix scaffolds provide a disease-specific environment for testing antifibrotic agents.

Pulmonary fibroblasts in IPF matrix scaffolds showed a mean growth rate (linear fit: slope $=6.74$, $\mathrm{R}^{2}=0.98$ ) over $80 \%$ faster than fibroblasts in normal lung matrix scaffolds (linear fit: slope $=3.70$, $R^{2}=0.93$; Fig. 6A, no drug), consistent with the fibroproliferative process characteristic of human IPF. To assess differences in phenotype between fibroblasts cultured on IPF matrix scaffolds and the conventional drug testing substrate tissue culture plastic, disease-associated gene expression and growth factor secretion were analyzed. Fibroblasts cultured in IPF matrix scaffolds expressed significantly higher COL1A1 and MMP2 than fibroblasts cultured on plastic (Fig. 6B), and secreted more profibrotic growth factors bFGF and TGF $\beta$ than fibroblasts cultured in normal lung matrix or on plastic (Fig. 6C,D), suggesting that the presence of disease-specific matrix resulted 357 in more disease-associated fibroblast phenotype in vitro compared to fibroblasts on plastic. When 358 exposed to antifibrotic agent PF3644022, a potent ATP-competitive MK2 inhibitor, pulmonary 359 fibroblasts cultured in IPF matrix scaffolds demonstrated significant reduction in cell number 360 compared to untreated fibroblasts over 6 days. PF3644022 also reduced expression of key IPF- 
A

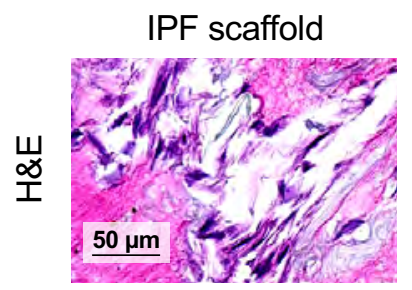

B

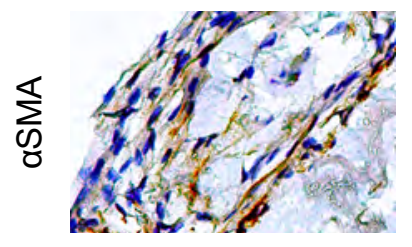

C

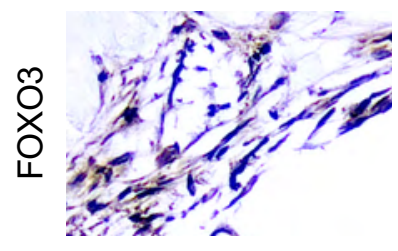

normal scaffold
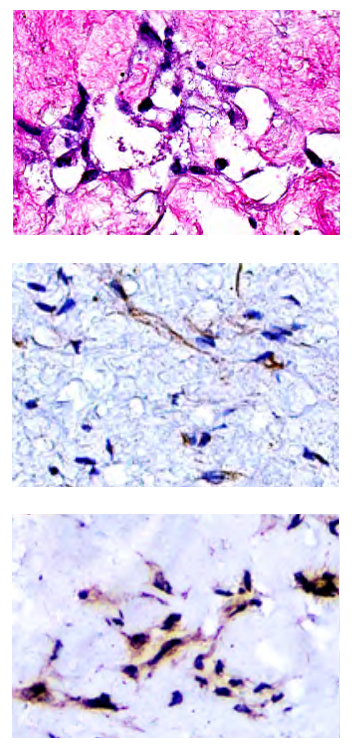
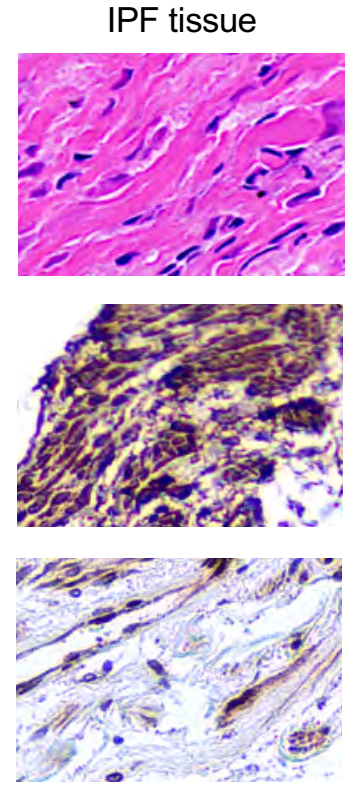

D

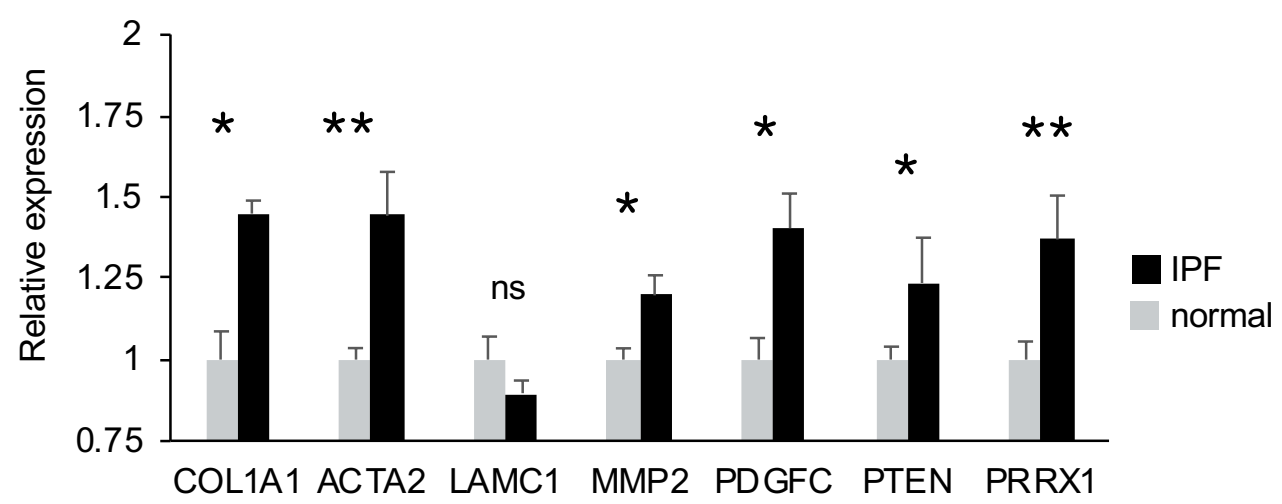

E

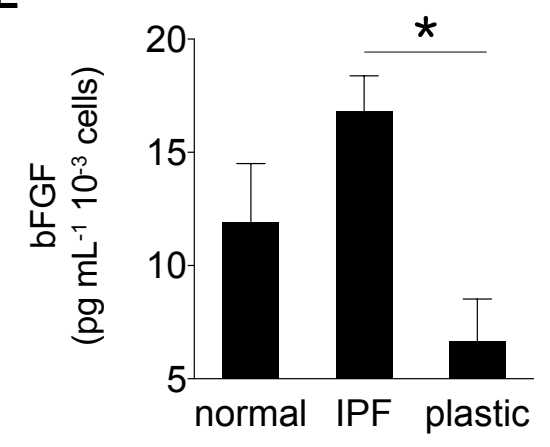

$\mathbf{F}$

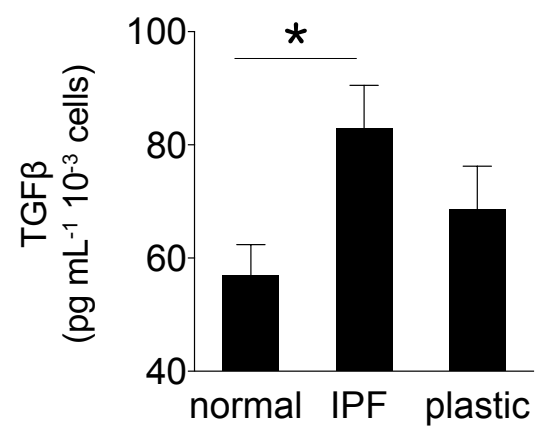

Figure 5 | Phenotype of lung fibroblasts in IPF and normal lung scaffolds. Representative micrographs of (A) H\&E and immunohistochemical staining of $(B)$ alpha smooth muscle actin (aSMA) and (C) Forkhead box O3 (FOXO3). (D) Gene expression of normal human lung fibroblasts cultured in IPF and normal lung scaffolds. ${ }^{*} p<0.05,{ }^{* *} p<0.01$, ns: not significant. Quantification by ELISA of (E) basic fibroblast growth factor (bFGF, ${ }^{*} p<0.05$ ) and $(\mathbf{F})$ transforming growth factor beta (TGF $\beta,{ }^{*} p<0.05$ ) secreted by normal human lung fibroblast cultured in IPF and normal lung scaffolds and on tissue culture plastic. All values represent mean \pm standard deviation. 


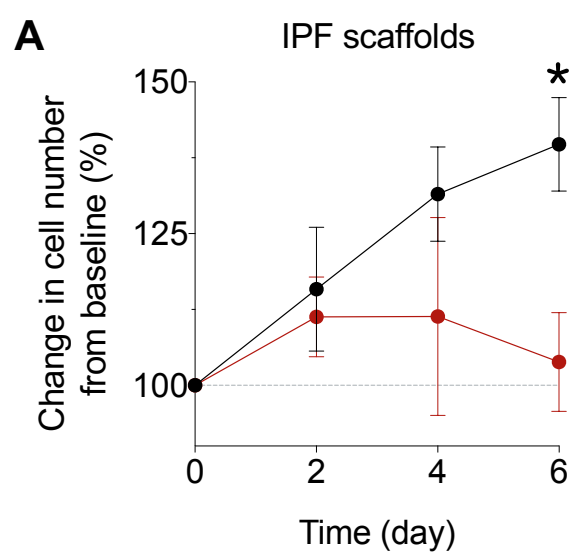

B
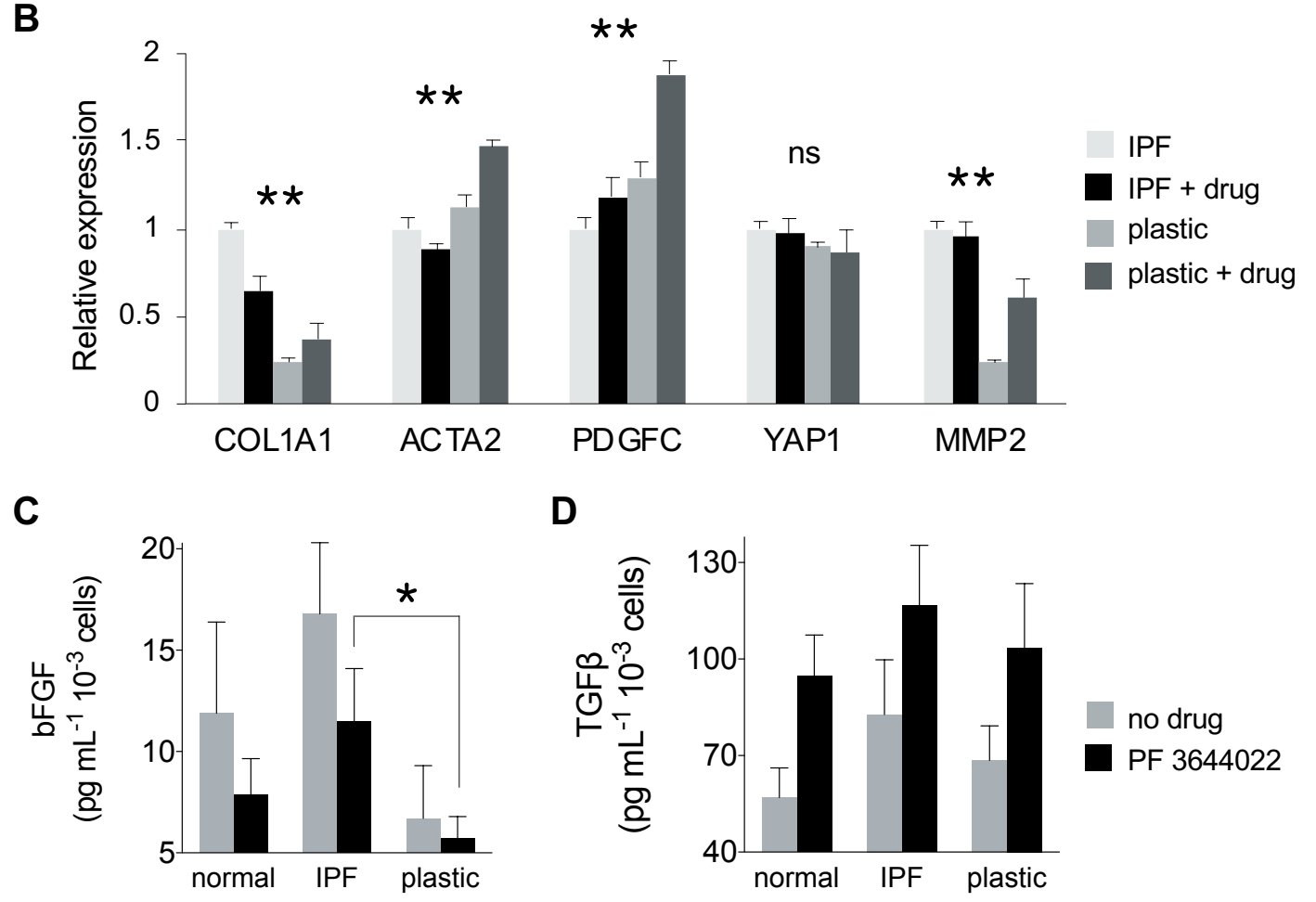

Figure 6 | Demonstration of anti-fibrotic drug testing in IPF lung scaffolds. (A) Growth curves of normal human lung fibroblasts over 6 days of treatment with PF 3644022. (B) Gene expression of normal human lung fibroblasts cultured on IPF scaffolds and tissue culture plastic. ${ }^{*} p<0.05,{ }^{* *} p<0.01$. Quantification by ELISA of (C) basic fibroblast growth factor (bFGF, $p=0.0024$ by ANOVA) and (D) transforming growth factor beta (TGF $\beta, p=0.0084$ by ANOVA) secreted by normal lung fibroblast cultured in IPF and normal lung scaffolds and on tissue culture plastic. All values represent mean \pm standard deviation. 
associated genes COL1A1 and ACTA2 by fibroblasts in IPF matrix scaffolds (Fig. 6B) - an expected result not observed in fibroblasts cultured on plastic. Similarly, PF3644022 reduced secretion of bFGF by fibroblasts cultured in IPF matrix scaffolds (Fig. 6C). Interestingly, secretion of TGF $\beta$ by fibroblasts exposed to PF3644022 trended upward across all substrates (Fig. 6D). Altogether, these results confirm the activation and diseased phenotype of pulmonary fibroblasts cultured in IPF matrix, and demonstrate the feasibility of testing antifibrotic agents in an in-vitro substrate environment with IPF disease-specific features not otherwise present in tissue culture plastic or other conventional drug screening platforms.

\section{DISCUSSION}

Using a physiomimetic approach, we developed an IPF disease-specific 3D cell culture substrate comprised of fibrotic human lung extracellular matrix. Through biomolecular and physicomechanical characterizations, we show that this disease-specific substrate has numerous physical and compositional features of the human IPF diseased extracellular matrix environment. We also demonstrate the applicability of this substrate for pharmaceutical drug testing. As the

377 critical need for effective IPF drugs persists, human IPF disease-specific cell culture substrates 378 could enable more predictive disease models and drug screening platforms, and accelerate 379 development of new drugs for the treatment of IPF.

381 Human IPF is a chronic, aging-related disease of unknown etiology typically diagnosed at an 382 advanced stage, and is therefore challenging to model. Both animal ${ }^{10,13}$ and in-vitro models ${ }^{18,19,35-}$ $383{ }^{37}$ have been used to gain insights into the cellular and molecular mechanisms of IPF. Although 384 animal models of IPF have been developed in mice, rats, hamsters, guinea pigs, rabbits, cats, 385 dogs, sheep, donkeys, horses, and non-human primates, ${ }^{11,38-40}$ no animal model fully 386 recapitulates the pathophysiology of human IPF - specifically, the histologic pattern of usual 
387 interstitial pneumonia and progressive fibrotic disease. ${ }^{13}$ Furthermore, while animal models may 388 inform various aspects of fibrotic lung disease, significant anatomical, biological, and 389 immunological differences from humans reduce pathophysiological relevance to human IPF. 390 Notably, the American Thoracic Society has emphasized the importance of developing 391 'humanized' models of IPF to increase relevance of animal models of IPF to the human disease. ${ }^{41}$

393 Because animal models of IPF are inherently limited, in-vitro models are an indispensable tool in 394 basic and translational studies of human IPF. Previous studies have implicated multiple cellular 395 processes in pulmonary fibrosis including epithelial cell apoptosis ${ }^{42}$, epithelial-mesenchymal 396 transition $^{43}$, and differentiation of fibroblasts to myofibroblasts ${ }^{44}$ that result in significant 397 remodeling and deposition of fibrotic ECM. Conventional two-dimensional (2D) models of IPF typically utilize monolayers of pulmonary myofibroblasts, the primary effector cells of IPF ${ }^{45}$, on tissue culture plastic, enabling mechanistic studies in controlled experimental settings. However, cells cultured in 2D models experience artificial, non-physiological conditions that categorically 401 lack the appropriate three-dimensional (3D) spatial gradients - chemical, mechanical, 402 topographical - in which all lung cells naturally reside within the body. Constrained to one spatial 403 plane, cells in 2D models are immobilized, experience limited cell-cell interactions, and display 404 inhibited cytokinesis and chemotaxis, artificial flattened morphology, unnatural apical-basal 405 polarization, and abnormal integrin and cell-surface receptor expression and distribution. ${ }^{46-48}$ 406 Furthermore, tissue culture plastic has non-physiological topography and stiffness $(>1,000$ $407 \mathrm{kPa})^{49}$, which has been shown to drive atypical cytoskeletal rearrangements ${ }^{50}$, perturb 408 homeostatic gene expression ${ }^{51}$, and induce epigenetic modifications of fibroblasts ${ }^{52}$.

410 Pulmonary fibroblasts cultured in 3D models, however, adhere to substrates at multiple focal 411 adhesion points ${ }^{53}$, and experience more in vivo-like stress-strain ${ }^{47}$ and soluble ${ }^{54}$ gradients. Lung 412 fibroblasts cultured in hydrogels of collagen type I, a structural ECM component upregulated in 
413 IPF, previously displayed contraction of collagen hydrogels, whose resistance to cell-generated

414 forces was proportional to expression of aSMA by fibroblasts. ${ }^{55}$ Notably, collagen type I hydrogels

415 are comprised of a single structural ECM component, and thus lack the complex signaling and

416 regulation of the multi-component ECM in the fibrotic disease environment. As paracrine, cell-

417 cell, and cell-matrix interactions are known to drive progression of fibrotic lung disease ${ }^{56}$ disease-

418 specific 3D cell culture substrates are critical for improving in-vitro models of IPF, and should

419 ideally recapitulate the structure, mechanics, and biochemical composition of diseased human

420 lung tissue.

422 In this study, biochemical and mass spectrometry analyses confirmed that IPF matrix scaffolds

423 had: (i) increased collagens and decreased elastin consistent with increased stiffness and

424 decreased compliance, (ii) increased proteoglycans, whose covalently bound glycosaminoglycan

425 side chains chondroitin sulfate, dermatan sulfate, heparan sulfate, and hyaluronic acid have been

426 shown to be structurally altered and increased in IPF lungs ${ }^{57}$, and (iii) abnormal profile of

427 glycoproteins. Proteoglycans influence viscoelastic properties, cell differentiation, and tissue

428 morphogenesis, and in particular heparan sulfate coordinates ligand-receptor binding of FGF,

429 PDGF, TGF $\beta$, and VEGF ${ }^{58}$ - growth factors involved in pathologic tissue remodeling and detected

430 in IPF matrix scaffolds. Biglycan, a small leucine-rich proteoglycan (SLRP) known to be altered in

431 fibrosis and correlated with lung mechanics through influence on ECM assembly ${ }^{59}$, was increased

432 over $600 \%$ in IPF matrix scaffolds. The perturbed profile of glycoproteins in IPF matrix scaffolds

433 included: increased fibrillin 2, a collagenase-resistant glycoprotein that is associated with the 10-

$434 \mathrm{~nm}$ microfibrils of the basal lamina and regulates the bioavailability of TGB $\beta$ through latent

435 transforming growth factor $\beta$ binding proteins $(\text { LTBP })^{60}$, and increased periostin, a matricellular

436 glycoprotein that promotes fibroblast proliferation, localization of fibrogenic growth factors,

437 collagen type I production, and collagen crosslinking ${ }^{61}$. Notably, the loss of basement membrane

438 components including collagen type IV ${ }^{29}$, laminin, and nidogen in IPF tissues and matrix scaffolds 
suggests that the use of basement membrane extracts such as Matrigel in models of IPF has

440 minimal pathophysiologic relevance.

442 The stiffness of fibrotic lung tissue $(60 \pm 40 \mathrm{kPa})$ is significantly higher than the stiffness of normal

443 lung tissue $(7 \pm 6 \mathrm{kPa})^{35,49}$, which has critical implications for the stiffness of cell culture substrates

444 in models of IPF, especially for in-vitro culture of pulmonary fibroblasts, which exhibit complex 445 mechanotransduction 20,62 and have 'mechanical memory'63. In this study, fibrotic human lung

446 ECM scaffolds recapitulated the mechanical differences between normal and fibrotic lung tissues

447 (Fig. 4), and supported increased secretion of bFGF and TGF $\beta$ by normal human lung fibroblasts

448 (Fig. 5E,F), suggesting that the IPF matrix scaffolds have disease-specific mechanics and

449 regulatory signals relevant to human IPF. Notably, pulmonary fibroblasts cultured in fibrotic lung

450 ECM previously confirmed the regulatory role of ECM in the activation of myofibroblasts in vitro ${ }^{36}$,

451 and demonstrated significant effects of substrate stiffness on fibroblast activation and

452 differentiation into myofibroblasts. Myofibroblast differentiation has also been shown to be driven

453 by increased ECM stiffness through mechanisms independent of TGF $\beta^{20}$. Interestingly, previous

454 studies wherein normal and IPF fibroblasts were cultured across ECM from normal and IPF lungs

455 revealed that IPF ECM had a greater influence on fibroblast gene expression than cell origin ${ }^{64}$,

456 further indicating the central role of ECM in regulating disease-associated gene expression.

457 Altogether, these results highlight the critical importance of providing disease-specific signals from

458 the ECM environment in models of fibrotic lung disease.

460 In spite of decades of basic and translational research, the persisting struggle to successfully 461 translate promising preclinical drug candidates to drugs approved to treat IPF highlights the 462 limited effectiveness of disease models used in IPF drug discovery, which is likely attributable to 463 the failure of IPF models to recapitulate key pathophysiological features of the human disease. 464 Early-stage drug discovery assays are typically conducted on tissue culture plastic (e.g., 
465 polystyrene) with or without collagen type I coating, and supplemental TGF $\beta$ (e.g., $1-5 \mathrm{ng} \mathrm{mL}^{-1}$ )

466 to activate primary or immortalized human lung fibroblasts - an entrenched in-vitro system that

467 has minimal pathophysiological relevance to the human IPF disease setting. With significant

468 financial costs and scientific, medical, and regulatory challenges associated with conducting

469 clinical trials in patients with IPF, preclinical assessments of antifibrotic compounds must be

470 sufficiently robust to inform go/no go decision making and yield reliably predictive data in order to

471 maximize the likelihood of advancing promising drug candidates to clinical trials.

472

473 In this study, we demonstrated the use of IPF disease-specific ECM in a 3D cell-based assay of

474 antifibrotic agent PF3644022 (an MK2 inhibitor in IPF model) ${ }^{65}$. As expected, fibroblasts cultured

475 on fibrotic lung ECM scaffolds and treated with PF3644022 exhibited greater sensitivity and drug

476 response, significantly different gene expression, and downregulation of genes associated with

477 ECM production compared to cells cultured on tissue culture plastic. We envision that disease-

478 specific ECM may be applicable across multiple stages of the drug discovery pipeline, from target

479 selection and hit identification through lead identification and optimization. The use of disease-

480 specific ECM substrates is consistent with the set of principles ${ }^{66}$ defined for 'disease-relevant

481 assays' that specifically recommend ensuring: (i) substrate tension and mechanical forces are

482 appropriate, and (ii) extracellular matrix composition is relevant, with appropriate tissue

483 architecture, cell differentiation and function to enhance clinical translation of the in-vitro assay.

484 Ultimately, implementation of disease-specific ECM components or substrates into preclinical

485 human disease models and cell-based screening assays could increase clinical relevance and 486 success rates.

488 There are several limitations to the present study: (1) This study investigated a small number of 489 human lungs ( $n=6$ total, $n=3$ per group). Although this study was conducted with the minimum 490 number of human lungs required to achieve statistical significance between groups, investigation 
491 of larger numbers of IPF lungs would offer opportunities for deeper statistical analyses and

492 potential correlations between matrix characteristics and disease phenotypes. (2) As IPF lung 493 specimens were procured from explanted tissues following lung transplantation, this study only 494 investigated fibrotic lung matrix from end-stage disease. While diagnosis of IPF remains a 495 significant clinical challenge, procurement of fibrotic human lung ECM at earlier stages of fibrotic 496 disease may not be feasible. (3) Human donor tissues present intrinsic biological variability that 497 could confound experimental results. To minimize variability between donors, acceptance criteria 498 for lungs were tightly defined and strictly implemented. Furthermore, as IPF is a disease with 499 demonstrable spatiotemporal heterogeneity, extensive histopathologic review was conducted by 500 a lung transplant pathologist to ensure only tissues and scaffolds with fibrosis scores $\geq 2$ were 501 utilized. (4) Only one antifibrotic drug was evaluated in this study. Future studies will investigate 502 additional compounds to provide further evidence of the utility and benefits of IPF disease-specific 503 ECM substrates.

505 The IPF matrix scaffolds developed in this study may be useful for cell-based assays, but may 506 have limited applicability to high throughput drug screening systems, which typically utilize rapid 507 optical readouts in 96- and 384-well plate formats. Therefore, an alternative format of fibrotic lung 508 matrix, e.g., hydrogel, may be more suitable for high throughput applications. Future studies will 509 explore the development of additional IPF disease-specific ECM formats to address broader 510 research and development applications such as 'IPF-on-chip'. As IPF disease progression is 511 driven by a combination of lung and immune cell-cell and cell-matrix interactions, future studies 512 will also investigate co-cultures with pulmonary macrophages, epithelial and smooth muscle cells, 513 and the effects of IPF-associated growth factors on lung cells in vitro. Altogether, an in-vitro model 514 with a disease-specific substrate of human IPF disease environment can help elucidate 515 underlying idiopathologies of IPF, enable development of effective IPF therapeutics, and may 
serve as a template approach for the development of fibrosis-specific cell culture substrates in

517 other human organs and tissues susceptible to fibrotic disease.

\section{CONCLUSIONS}

520 We developed a pulmonary fibrosis-specific cell culture substrate comprised of intact fibrotic lung

521 extracellular matrix that recapitulated in vitro key features of the human IPF disease environment

522 and supported the disease-associated phenotype of human lung fibroblasts. We also

523 demonstrated feasibility of testing antifibrotic agents using this substrate, which may be applicable

524 in cell-based assays in early-stage drug discovery.

\section{ACKNOWLEDGEMENTS}

527 The authors would like to thank M. Bacchetta and G. Vunjak-Novakovic for discussions of the 528 experimental design and results; M. Bacchetta and Y. Tipograf for coordinating receipt of human 529 lung tissues; Albert Einstein College of Medicine Analytical Imaging Facility for slide scanning; C.

530 Marboe for conducting blinded pathologic assessments; and Weill Cornell Microscopy and Image 531 Analysis Core Facility staff, including L. Cohen-Gould and J. P. Jimenez for transmission electron 532 microscopy imaging services. The authors gratefully acknowledge funding support 533 (1R43HL144341) from the National Institutes of Health.

\section{AUTHOR CONTRIBUTIONS}

536 I.G., E.A., and J.D.O. designed the study. I.G., E.A., J. X., N. K., A. N., and E.G. performed 537 experiments. I.G., E.A., and J.D.O. co-analyzed data, co-wrote the manuscript. 


\section{REFERENCES}

539

5401 Lederer, D. J. \& Martinez, F. J. Idiopathic Pulmonary Fibrosis. N Engl J Med 378, 1811-

541 1823, doi:10.1056/NEJMra1705751 (2018).

5422 Coward, W. R., Saini, G. \& Jenkins, G. The pathogenesis of idiopathic pulmonary fibrosis.

543 Ther Adv Respir Dis 4, 367-388, doi:10.1177/1753465810379801 (2010).

5443 Noble, P. W., Barkauskas, C. E. \& Jiang, D. Pulmonary fibrosis: patterns and perpetrators.

$545 \quad J$ Clin Invest 122, 2756-2762, doi:10.1172/JCI60323 (2012).

5464 Sauleda, J., Nunez, B., Sala, E. \& Soriano, J. B. Idiopathic Pulmonary Fibrosis:

547 Epidemiology, Natural History, Phenotypes. Med Sci (Basel) 6,

548 doi:10.3390/medsci6040110 (2018).

5495 Ley, B. \& Collard, H. R. Epidemiology of idiopathic pulmonary fibrosis. Clin Epidemiol 5,

550 483-492, doi:10.2147/CLEP.S54815 (2013).

5516 Hutchinson, J., Fogarty, A., Hubbard, R. \& McKeever, T. Global incidence and mortality of

552 idiopathic pulmonary fibrosis: a systematic review. Eur Respir J 46, 795-806,

553 doi:10.1183/09031936.00185114 (2015).

5547 Vancheri, C., Failla, M., Crimi, N. \& Raghu, G. Idiopathic pulmonary fibrosis: a disease with 555 similarities and links to cancer biology. Eur Respir J 35, 496-504,

556 doi:10.1183/09031936.00077309 (2010).

5578 Ryu, J. H. et al. Idiopathic pulmonary fibrosis: evolving concepts. Mayo Clin Proc 89, 1130$558 \quad$ 1142, doi:10.1016/j.mayocp.2014.03.016 (2014).

5599 Plantier, L. et al. Physiology of the lung in idiopathic pulmonary fibrosis. Eur Respir Rev 27, 560 doi:10.1183/16000617.0062-2017 (2018).

56110 Moore, B. B. \& Hogaboam, C. M. Murine models of pulmonary fibrosis. Am J Physiol Lung

562 Cell Mol Physiol 294, L152-160, doi:10.1152/ajplung.00313.2007 (2008). 
56311 Mouratis, M. A. \& Aidinis, V. Modeling pulmonary fibrosis with bleomycin. Curr Opin Pulm

$564 \quad$ Med 17, 355-361, doi:10.1097/MCP.0b013e328349ac2b (2011).

56512 Antje Moeller, J. C. R.-L., Lingqiao Wang, Jack Gauldie, Martin Kolb. Models of pulmonary

566 fibrosis. Drug Discovery Today Disease Models 3, 243-249 (2006).

56713 B, B. M. et al. Animal models of fibrotic lung disease. Am J Respir Cell Mol Biol 49, 167-

$568 \quad$ 179, doi:10.1165/rcmb.2013-0094TR (2013).

56914 Nichols, J. E., Niles, J. A., Vega, S. P. \& Cortiella, J. Novel in vitro respiratory models to

570 study lung development, physiology, pathology and toxicology. Stem Cell Res Ther 4

$571 \quad$ Suppl 1, S7, doi:10.1186/scrt368 (2013).

57215 Zhou, Y. et al. Extracellular matrix in lung development, homeostasis and disease. Matrix

573 Biol 73, 77-104, doi:10.1016/j.matbio.2018.03.005 (2018).

57416 Shimbori, C., Gauldie, J. \& Kolb, M. Extracellular matrix microenvironment contributes

575 actively to pulmonary fibrosis. Curr Opin Pulm Med 19, 446-452,

576 doi:10.1097/MCP.0b013e328363f4de (2013).

57717 Kristensen, J. H. et al. The role of extracellular matrix quality in pulmonary fibrosis.

$578 \quad$ Respiration 88, 487-499, doi:10.1159/000368163 (2014).

57918 Hetzel, M., Bachem, M., Anders, D., Trischler, G. \& Faehling, M. Different effects of growth 580 factors on proliferation and matrix production of normal and fibrotic human lung fibroblasts.

$581 \quad$ Lung 183, 225-237, doi:10.1007/s00408-004-2534-z (2005).

58219 Correll, K. A. et al. TGF beta inhibits HGF, FGF7, and FGF10 expression in normal and IPF 583 lung fibroblasts. Physiol Rep 6, e13794, doi:10.14814/phy2.13794 (2018).

58420 Huang, X. et al. Matrix stiffness-induced myofibroblast differentiation is mediated by 585 intrinsic mechanotransduction. Am J Respir Cell Mol Biol 47, 340-348, 586 doi:10.1165/rcmb.2012-00500C (2012). 
21 Wight, T. N. \& Potter-Perigo, S. The extracellular matrix: an active or passive player in fibrosis? Am J Physiol Gastrointest Liver Physiol 301, G950-955, doi:10.1152/ajpgi.00132.2011 (2011).

22 Lehtonen, S. T. et al. Pirfenidone and nintedanib modulate properties of fibroblasts and myofibroblasts in idiopathic pulmonary fibrosis. Respir Res 17, 14, doi:10.1186/s12931016-0328-5 (2016).

23 O'Neill, J. D. et al. Decellularization of human and porcine lung tissues for pulmonary tissue engineering. Ann Thorac Surg 96, 1046-1055; discussion 1055-1046, doi:10.1016/j.athoracsur.2013.04.022 (2013).

24 Wagner, D. E. et al. Three-dimensional scaffolds of acellular human and porcine lungs for

25 Balestrini, J. L. et al. Comparative biology of decellularized lung matrix: Implications of species mismatch in regenerative medicine. Biomaterials 102, 220-230, doi:10.1016/j.biomaterials.2016.06.025 (2016). tissue regeneration. Eur Respir Rev 27, doi:10.1183/16000617.0021-2018 (2018). pulmonary fibrosis on a numerical scale. J Clin Pathol 41, 467-470,

60728 Lu, J., Auduong, L., White, E. S. \& Yue, X. Up-regulation of heparan sulfate 6-O-sulfation in 608 idiopathic pulmonary fibrosis. Am J Respir Cell Mol Biol 50, 106-114, 609 doi:10.1165/rcmb.2013-02040C (2014).

61029 Sand, J. M. et al. MMP mediated degradation of type IV collagen alpha 1 and alpha 3 611 chains reflects basement membrane remodeling in experimental and clinical fibrosis-- 
validation of two novel biomarker assays. PLoS One 8, e84934, doi:10.1371/journal.pone.0084934 (2013).

61430 S. Ohshimo, K. H., F. Bonella, C. Yamaoka, Y. Horimasu, H. Iwamoto, N. Ishikawa, K.

615 Fujitaka, H. Murai, H. Hamada, N. Hattori, N. Hirohashi, K. Tanigawa, J. Guzman, U.

616 Costabel, N. Kohno. Growth Differentiation Factor-15 (gdf-15) As Prognostic Factor For

617 Idiopathic Pulmonary Fibrosis. Am J Respir Crit Care Med 191 (2015).

61831 Ruan, W. \& Ying, K. Abnormal expression of IGF-binding proteins, an initiating event in 619 idiopathic pulmonary fibrosis? Pathol Res Pract 206, 537-543,

620 doi:10.1016/j.prp.2010.03.010 (2010).

62132 Pilewski, J. M., Liu, L., Henry, A. C., Knauer, A. V. \& Feghali-Bostwick, C. A. Insulin-like 622 growth factor binding proteins 3 and 5 are overexpressed in idiopathic pulmonary fibrosis 623 and contribute to extracellular matrix deposition. Am J Pathol 166, 399-407, 624 doi:10.1016/S0002-9440(10)62263-8 (2005).

62533 Avcuoglu, S. et al. Neurotrophic tyrosine kinase receptor B/neurotrophin 4 signaling axis is 626 perturbed in clinical and experimental pulmonary fibrosis. Am J Respir Cell Mol Biol 45, 627 768-780, doi:10.1165/rcmb.2010-01950C (2011).

62834 Al-Tamari, H. M. et al. FoxO3 an important player in fibrogenesis and therapeutic target for 629 idiopathic pulmonary fibrosis. EMBO Mol Med 10, 276-293, 630 doi:10.15252/emmm.201606261 (2018).

63135 Hinz, B. Mechanical aspects of lung fibrosis: a spotlight on the myofibroblast. Proc Am 632 Thorac Soc 9, 137-147, doi:10.1513/pats.201202-017AW (2012).

63336 Booth, A. J. et al. Acellular normal and fibrotic human lung matrices as a culture system for 634 in vitro investigation. Am J Respir Crit Care Med 186, 866-876, doi:10.1164/rccm.201204$635 \quad 07540 C$ (2012). 
63637 Thannickal, V. J. et al. Matrix biology of idiopathic pulmonary fibrosis: a workshop report of 637 the national heart, lung, and blood institute. Am J Pathol 184, 1643-1651, 638 doi:10.1016/j.ajpath.2014.02.003 (2014).

63938 Tashiro, J. et al. Exploring Animal Models That Resemble Idiopathic Pulmonary Fibrosis.

$640 \quad$ Front Med (Lausanne) 4, 118, doi:10.3389/fmed.2017.00118 (2017).

64139 Paul F. Mercer, K. A.-B., lan M. Adcock, Richard G. Knowles. Translational models of lung 642 disease. Clinical Science 128 235-256, doi:10.1042/CS20140373 (2015).

64340 Organ, L. et al. A novel segmental challenge model for bleomycin-induced pulmonary $644 \quad$ fibrosis in sheep. Exp Lung Res 41, 115-134, doi:10.3109/01902148.2014.985806 (2015).

64541 R. Gisli Jenkins, B. B. M., Rachel C. Chambers, Oliver Eickelberg, Melanie Konigshoff, 646 Martin Kolb, Geoffrey J. Laurent, Carmel B. Nanthakumar, Mitchell A. Olman, Annie Pardo, Moises Selman, Dean Sheppard, Patricia J. Sime, Andrew M. Tager, Amanda L. Tatler,

42 Lepparanta, O. et al. Regulation of TGF-beta storage and activation in the human idiopathic pulmonary fibrosis lung. Cell Tissue Res 348, 491-503, doi:10.1007/s00441-012-1385-9 (2012).

$43 \mathrm{Kim}, \mathrm{K}$. K. et al. Alveolar epithelial cell mesenchymal transition develops in vivo during pulmonary fibrosis and is regulated by the extracellular matrix. Proc Natl Acad Sci U S A

44 Kis, K., Liu, X. \& Hagood, J. S. Myofibroblast differentiation and survival in fibrotic disease. Expert Rev Mol Med 13, e27, doi:10.1017/S1462399411001967 (2011). 
66246 Sundarakrishnan, A., Chen, Y., Black, L. D., Aldridge, B. B. \& Kaplan, D. L. Engineered cell 663 and tissue models of pulmonary fibrosis. Adv Drug Deliv Rev 129, 78-94, 664 doi:10.1016/j.addr.2017.12.013 (2018).

66547 Baker, B. M. \& Chen, C. S. Deconstructing the third dimension: how 3D culture 666 microenvironments alter cellular cues. J Cell Sci 125, 3015-3024, doi:10.1242/jcs.079509 667 (2012).

66848 Griffith, L. G. \& Swartz, M. A. Capturing complex 3D tissue physiology in vitro. Nat Rev Mol 669 Cell Biol 7, 211-224, doi:10.1038/nrm1858 (2006).

67049 Skardal, A., Mack, D., Atala, A. \& Soker, S. Substrate elasticity controls cell proliferation, 671 surface marker expression and motile phenotype in amniotic fluid-derived stem cells. $J$ 672 Mech Behav Biomed Mater 17, 307-316, doi:10.1016/j.jmbbm.2012.10.001 (2013).

67350 Doyle, A. D. \& Yamada, K. M. Mechanosensing via cell-matrix adhesions in 3D microenvironments. Exp Cell Res 343, 60-66, doi:10.1016/j.yexcr.2015.10.033 (2016).

67551 Raab, M., Shin, J. W. \& Discher, D. E. Matrix elasticity in vitro controls muscle stem cell fate in vivo. Stem Cell Res Ther 1, 38, doi:10.1186/scrt38 (2010).

67752 Nestor, C. E. et al. Rapid reprogramming of epigenetic and transcriptional profiles in 678 mammalian culture systems. Genome Biol 16, 11, doi:10.1186/s13059-014-0576-y (2015).

67953 Lou, J., Stowers, R., Nam, S., Xia, Y. \& Chaudhuri, O. Stress relaxing hyaluronic acid680 collagen hydrogels promote cell spreading, fiber remodeling, and focal adhesion formation 681 in 3D cell culture. Biomaterials 154, 213-222, doi:10.1016/j.biomaterials.2017.11.004 682 (2018).

68354 Klingberg, F. et al. Prestress in the extracellular matrix sensitizes latent TGF-beta1 for 684 activation. J Cell Biol 207, 283-297, doi:10.1083/jcb.201402006 (2014).

68555 Arora, P. D., Narani, N. \& McCulloch, C. A. The compliance of collagen gels regulates 686 transforming growth factor-beta induction of alpha-smooth muscle actin in fibroblasts. Am J 687 Pathol 154, 871-882, doi:10.1016/s0002-9440(10)65334-5 (1999). 
68856 Barkauskas, C. E. \& Noble, P. W. Cellular mechanisms of tissue fibrosis. 7. New insights 689 into the cellular mechanisms of pulmonary fibrosis. Am J Physiol Cell Physiol 306, C9876909 996, doi:10.1152/ajpcell.00321.2013 (2014).

69157 Westergren-Thorsson, G. et al. Increased deposition of glycosaminoglycans and altered 692 structure of heparan sulfate in idiopathic pulmonary fibrosis. Int J Biochem Cell Biol 83, 27$693 \quad 38$, doi:10.1016/j.biocel.2016.12.005 (2017).

69458 Forsten-Williams, K., Chu, C. L., Fannon, M., Buczek-Thomas, J. A. \& Nugent, M. A. 695 Control of growth factor networks by heparan sulfate proteoglycans. Ann Biomed Eng 36, 696 2134-2148, doi:10.1007/s10439-008-9575-z (2008).

69759 Faffe, D. S. \& Zin, W. A. Lung parenchymal mechanics in health and disease. Physiol Rev $698 \quad 89,759-775$, doi:10.1152/physrev.00019.2007 (2009).

69960 Davis, M. R. \& Summers, K. M. Structure and function of the mammalian fibrillin gene 700 family: implications for human connective tissue diseases. Mol Genet Metab 107, 635-647, 701 doi:10.1016/j.ymgme.2012.07.023 (2012).

70261 O'Dwyer, D. N. \& Moore, B. B. The role of periostin in lung fibrosis and airway remodeling. 703 Cell Mol Life Sci 74, 4305-4314, doi:10.1007/s00018-017-2649-z (2017).

70462 Branco da Cunha, C. et al. Influence of the stiffness of three-dimensional alginate/collagen705 I interpenetrating networks on fibroblast biology. Biomaterials 35, 8927-8936, 706 doi:10.1016/j.biomaterials.2014.06.047 (2014).

70763 Balestrini, J. L., Chaudhry, S., Sarrazy, V., Koehler, A. \& Hinz, B. The mechanical memory 708 of lung myofibroblasts. Integrative Biology 4, 410-421, doi:10.1039/c2ib00149g (2012).

70964 Parker, M. W. et al. Fibrotic extracellular matrix activates a profibrotic positive feedback $710 \quad$ loop. J Clin Invest 124, 1622-1635, doi:10.1172/JCI71386 (2014).

71165 Mourey, R. J. et al. A benzothiophene inhibitor of mitogen-activated protein kinase712 activated protein kinase 2 inhibits tumor necrosis factor alpha production and has oral anti- 
713 inflammatory efficacy in acute and chronic models of inflammation. $J$ Pharmacol Exp Ther

$714 \quad 333,797-807$, doi:10.1124/jpet.110.166173 (2010).

71566 Horvath, P. et al. Screening out irrelevant cell-based models of disease. Nat Rev Drug

716 Discov 15, 751-769, doi:10.1038/nrd.2016.175 (2016).

717 
Figure 1 | Overview of physiomimetic approach. Our development of IPF disease-specific cell

721 culture substrates is guided by a physiomimetic approach that aims to identify and isolate the

722 human disease environment, then develop and investigate disease-specific ECM substrates in

723 vitro utilizing disease-relevant human cell types (e.g., pulmonary fibroblasts) whose phenotype

724 can be directly compared against diseased human IPF lung specimens prior to application in IPF

725 disease models and antifibrotic drug testing.

726

727 Figure 2 | Histological \& biochemical characterization of ECM structural components in

728 IPF lung tissues and matrix scaffolds. Representative micrographs of histologic stains: (A)

729 hematoxylin and eosin (H\&E), (B) trichrome (collagens, blue), and (C) Verhoeff-Van Gieson

730 (VVG, elastic fibers, black) demonstrating differences in histomorphology of IPF and normal lung

731 tissues and scaffolds. Star indicates representative region with severe fibrosis and loss of elastic

732 fibers. Quantification of structural ECM components (D) collagens and (E) elastin by biochemical

733 assays. (F) Changes from normal lung in structural ECM components in IPF. H\&E: hematoxylin

734 and eosin, VVG: Verhoeff-Van Gieson.

735

736 Figure 3 | Characterization of proteoglycans and glycoproteins in IPF lung tissues and

737 matrix scaffolds. Representative micrographs of histologic stains: (A) Alcian blue

738 (proteoglycans, blue) and (B) pentachrome (acidic polysaccharides, green) demonstrating

739 differences in proteoglycans between IPF and normal lung tissues. Arrow indicates normal airway

740 epithelium. Quantification of sulfated glycosaminoglycan ECM components in (C) native tissues

741 and (D) matrix scaffolds. (E) Changes from normal lung in glycosaminoglycan ECM components

742 in IPF. Immunohistochemical staining of glycoprotein ECM components in IPF: (F) fibrillin 2, (G) 
743 laminin $\mathrm{Y1},(\mathbf{H})$ matrix gla protein (MGP), (I) periostin. (J) Quantification of glycoproteins by image

744 analysis of immunohistochemical staining using DensitoQuant software.

745

746 Table 1 | Mass spectrometry analysis of IPF lung matrisome. Changes from normal in

747 the abundance of IPF lung matrisome components. PG: proteoglycan, BM: basement membrane.

Table 2 | Quantification of growth factors in IPF and normal lung matrix scaffolds. Growth factor concentrations were measured by multiplex growth factor array. Green arrow $(\Delta)$ indicates positive fold change (increase) from normal in concentration of growth factors. Red arrow $(\nabla)$ indicates negative fold change (decrease) from normal in concentration of growth factors. ND: not detected.

Figure 4 | Structural, topographical, and mechanical characterization of IPF lung scaffolds. Representative images of IPF and normal lung scaffolds: (A) gross photography,

(B) scanning electron microscopy, (C) light microscopy (inverted color micrograph) of trichome staining demonstrating topography of ECM fibers in IPF scaffolds, (D) transmission electron microscopy. A: airspace, C: alveolar capillary, E: elastin bundle fragments, F: fibroconnective collagenous matrix, arrow: basement membrane. (E) Representative uniaxial stress-strain curves of IPF and normal lung tissues and matrix scaffolds. (F) Change in uniaxial mechanical stress from normal lung tissues and matrix scaffolds. (G) Tangent modulus values. Statistical analyses

763 between tissues and scaffolds were performed using Student's $t$-test, with significance when $p<$

764 0.05. All values represent mean \pm standard deviation.

766 Figure 5 | Phenotype of lung fibroblasts in IPF and normal lung scaffolds. Representative 767 micrographs of $(\mathbf{A}) \mathrm{H} \& E$ and immunohistochemical staining of (B) alpha smooth muscle actin 768 (aSMA) and (C) Forkhead box O3 (FOXO3). (D) Gene expression of normal human lung 
fibroblasts cultured in IPF and normal lung scaffolds. ${ }^{*} p<0.05,{ }^{* *} p<0.01$, ns:

770 not significant. Quantification by ELISA of (E) basic fibroblast growth factor (bFGF, * $p<0.05)$

771 and (F) transforming growth factor beta (TGF $\left.\beta,{ }^{*} p<0.05\right)$ secreted by normal human lung

772 fibroblast cultured in IPF and normal lung scaffolds and on tissue culture plastic. All values

773 represent mean \pm standard deviation.

774

775 Figure 6 | Demonstration of antifibrotic drug testing in IPF lung scaffolds. (A) Growth curves

776 of normal human lung fibroblasts over 6 days of treatment with PF3644022. (B) Gene expression

777 of normal human lung fibroblasts cultured on IPF scaffolds and tissue culture plastic. ${ }^{*} p<0.05$,

$778^{* *} p<0.01$. Quantification by ELISA of $(\mathbf{C})$ basic fibroblast growth factor (bFGF, $p=0.0024$ by

779 ANOVA) and (D) transforming growth factor beta (TGF $\beta, p=0.0084$ by ANOVA) secreted by

780 normal lung fibroblast cultured in IPF and normal lung scaffolds and on tissue culture plastic. All

781 values represent mean \pm standard deviation

782

783 Supplementary Figure 1 | Characterization of human IPF and normal lung tissues. (A)

784 Donor characteristics of IPF and normal lung tissues. (B) Representative chest radiographs of

785 IPF and normal lung donors. Arrows indicate changed lung shape, decreased lung volume, 786 and increased radiopacity consistent with pulmonary fibrosis. (C) Fibrosis scoring rubric used to 787 assess the extent of architectural disruption and fibrosis in human lung tissues. (D) Overview of 788 the description and quantity of tissue samples analyzed in this study. Samples were collected 789 from three regions (i.e., medial, lateral, peripheral) of right middle lobes. (F) Demonstration of 790 quantitative image analysis method used for fibrosis scoring. For each fibrosis score, a weighted 791 average is calculated from the ratios of the total area. Five high-power fields were analyzed per 792 region, and an average fibrosis score was calculated for each region. (G) Fibrosis scores of 793 all regions of IPF and normal lungs investigated in this study. Only regions of IPF lungs with 794 fibrosis score $\geq 2$ (red triangle) were investigated in this study. ${ }^{*} p<0.001$. 
795 Supplementary Figure 2 | Quality control assays of IPF and normal lung

796 matrix scaffolds. Quantification of $(\mathbf{A})$ DNA to confirm removal of nuclear material from IPF

797 and normal lung matrix scaffolds. (B) Results of sterility and mycoplasma assays. Prior to use in

798 studies, IPF and normal lung matrix scaffolds were tested for absence of bacteria and fungi.

799 Scaffolds were also tested for absence of mycoplasma using MycoAlert PLUS Mycoplasma

800 Detection Assay. * $p<0.001$.

801

802 Supplementary Table 1 | Antibodies and ELISA kits.

803

804 Supplementary Table 2 | Primers.

805

806 Supplementary Table 3 | Demographic and clinical characteristics of human lung donors.

807 UIP, usual interstitial pneumonia.

808

809 Supplementary Table 4 | Quantification of growth factors in IPF and normal lung tissues.

810 Growth factor concentrations were measured by multiplex growth factor array. Green arrow ( $\Delta)$

811 indicates positive fold change (increase) from normal in concentration of growth factors. Red

812 arrow $(\nabla)$ indicates negative fold change (decrease) from normal in concentration of growth

813 factors. ND: not detected. * IPF-specific growth factor not detected in normal lung tissue. 


\section{SUPPLEMENTARY INFORMATION}

816 Characterization of human IPF and normal lung tissues. Donor characteristics of IPF and

817 normal lung tissues were analyzed to confirm that there were no significant differences in age,

818 height, weight, body mass index, or smoking history (Supplementary Fig. 1A, Supplementary

819 Table 3). The mean lung allocation score for IPF donors was $55.0 \pm 25.1$, which was the only

820 significant difference between IPF and normal lung donors $(p<0.05)$. Chest radiographs of lung

821 donors confirmed absence of apparent injury or underlying disease in normal lungs, and enabled

822 assessment of the extent and distribution of pulmonary fibrosis in IPF lungs. In contrast to normal

823 lungs, which appeared radiolucent and aerated, IPF lungs displayed a morphologic pattern of

824 usual interstitial pneumonia consistent with IPF and marked by diffuse radiopacities, reticulation,

825 architectural distortion, and honeycombing, especially in peripheral and basal regions

826 (Supplementary Fig. 1B).

827

828 In order to characterize the histopathology of all tissues investigated, a fibrosis scoring rubric was

829 used to assess the extent of architectural disruption and fibrosis (Supplementary Fig. 1C).

830 Samples were systematically collected from the medial and lateral regions of the right middle and

831 right lower lobes. Histologic samples were evaluated by light microscopy, and fibrosis scores were

832 assigned and averaged across five high-power fields. All high-power fields were subjected to

833 imaging analyses to quantify the relative areas corresponding to each fibrosis score

834 (Supplementary Fig. 1F). To ensure to the maximum possible extent a consistent degree of

835 pulmonary fibrosis across all samples, only samples with average fibrosis score $\geq 2$ (moderate or

836 severe fibrosis) were investigated in this study (Supplementary Fig. 1G).

838 Mass spectrometry. Protein profiling included short gel SDS-PAGE, in-gel digestion with trypsin, 839 and 2 hours LC-MS/MS. Samples were weighed and suspended in $130 \mu \mathrm{L}$ of $2.0 \%$ modified RIPA 
840 buffer with $1.6 \mathrm{~mm}$ stainless steel beads. Samples were homogenized in a Next Advance Bullet

841 Blender for 3 minutes at speed 10 , then heated at $100^{\circ} \mathrm{C}$ for 30 minutes. Samples were then

842 sonicated and clarified by centrifugation. The protein concentration of the extract was determined

843 using Qubit fluorometry (Life Technologies). Each sample (10 $\mu \mathrm{g})$ was processed by $2 \mathrm{~cm}$ SDS-

844 PAGE using a 10\% Bis-Tris NuPAGE Novex mini gel (ThermoFisher) with the MES buffer system.

845 The mobility region was excised and processed by in-gel digestion with trypsin using a ProGest

846 robot (DigiLab) with the following protocol: (1) Wash with $25 \mathrm{mM}$ ammonium bicarbonate followed

847 by acetonitrile. (2) Reduce with $10 \mathrm{mM}$ dithiothreitol at $60^{\circ} \mathrm{C}$ followed by alkylation with $50 \mathrm{mM}$

848 iodoacetamide at room temperature. (3) Digest with sequencing grade trypsin (Promega) at $37^{\circ} \mathrm{C}$

849 for 4 hours. (4) Quench with formic acid and analyzed without further processing.

Half of each digest was analyzed by nano LC-MS/MS with a Waters NanoAcquity HPLC system

interfaced to a mass spectrometer (Fusion Lumos, ThermoFisher). Peptides were loaded on a

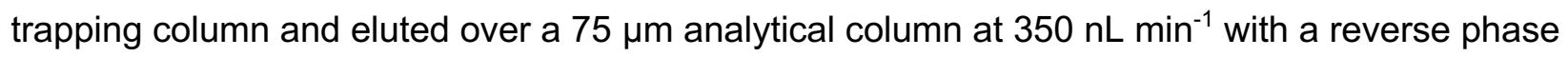
gradient for 2 hours. Both columns were packed with Luna C18 resin (Phenomenex). The mass spectrometer was operated in data-dependent mode, with the Orbitrap operating at 60,000 FWHM and 15,000 FWHM for MS and MS/MS, respectively. The instrument was run with a 3 second cycle for MS and MS/MS. Advanced Precursor Determination was employed. Data were searched using a local copy of Mascot (Matrix Science) with the following parameters: Enzyme: Trypsin/P; Database: SwissProt Human (concatenated forward and reverse plus common 860 contaminants); Fixed modification: Carbamidomethyl (C); Variable modifications: Oxidation (M/P), 861 Acetyl (N-term), Pyro-Glu (N-term Q), Deamidation (N,Q); Mass values: Monoisotopic; Peptide 862 Mass Tolerance: 10 ppm; Fragment Mass Tolerance: 0.02 Da; Max Missed Cleavages: 2. Mascot 863 DAT files were parsed into Scaffold (Proteome software) for validation and filtering to create a 864 non-redundant list per sample. Data were filtered using at least $1 \%$ protein and peptide FDR, and 865 requiring at least two unique peptides per protein. 
A

\begin{tabular}{llll} 
Lung donor characteristics & IPF & normal & p-value \\
\hline Age (years) & $61 \pm 6$ & $44 \pm 17$ & 0.18 \\
Height $(\mathrm{cm})$ & $160 \pm 5$ & $173 \pm 7$ & 0.06 \\
Weight $(\mathrm{kg})$ & $55 \pm 12$ & $81 \pm 18$ & 0.10 \\
BMI $\left(\mathrm{kg} \mathrm{m}^{-2}\right)$ & $21 \pm 3$ & $27 \pm 6$ & 0.22 \\
Smoking history (pack years) & 0 & 0 & - \\
Lung allocation score & $55.0 \pm 25.1$ & 0 & $\mathbf{0 . 0 2}$
\end{tabular}

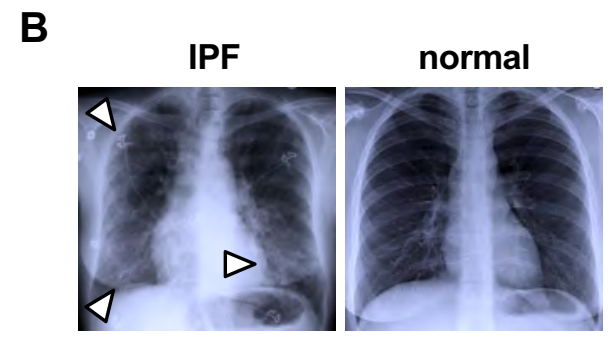

C Classification

\section{Representative H\&E staining}

Fibrosis score

Histologic
features

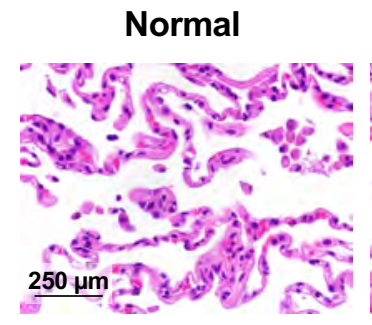

0

Normal lung or minimal fibrous thickening of bronchiolar walls or alveolar septa

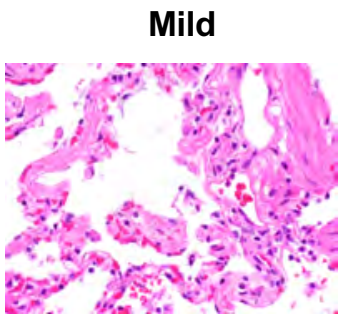

1

Moderate thickening of walls or septa without obvious damage to lung architecture

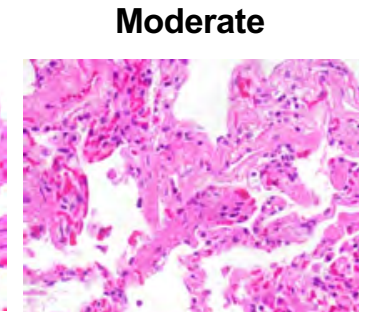

2

Increased fibrosis with damage to lung structure and formation of fibrous bands or masses
Severe

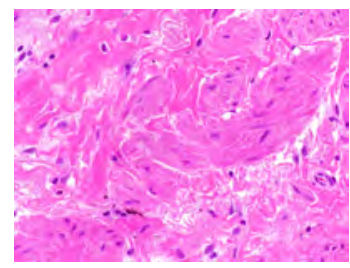

3

Severe distortion of structure and large fibrous areas; "honeycomb lung"; or total fibrous obliteration

D

\begin{tabular}{llc} 
Analyzed in this study & Description & Quantity \\
\hline Lungs & IPF & 3 \\
& normal & 3 \\
Lobes per lung & right middle lobe (RML) & 1 \\
Regions per right middle lobe & medial, lateral, peripheral & 3 \\
Samples per region & medial $\times 2$, lateral $\times 2$, peripheral $\times 2$ & 2 \\
Samples per lung & 2 samples per region $\times 3$ regions & 6 \\
\hline Total IPF lung samples & 6 samples per RML $\times 3$ lungs & $\mathbf{1 8}$ \\
Total normal lung samples & 6 samples per RML $\times 3$ lungs & $\mathbf{1 8}$
\end{tabular}

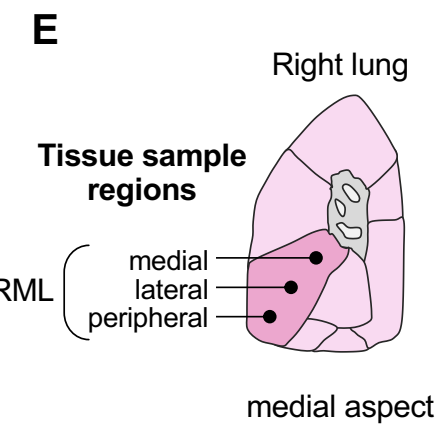

$\mathbf{F}$

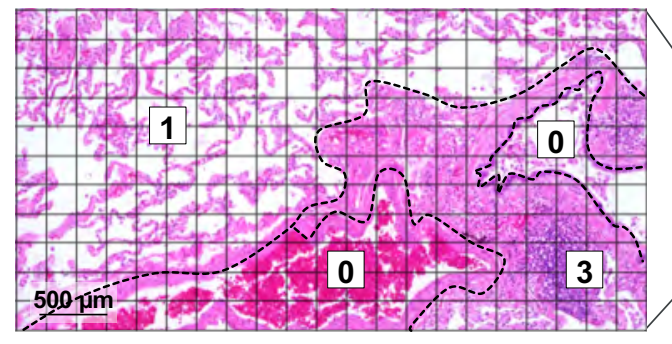

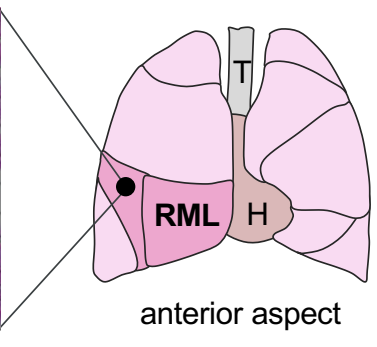

G

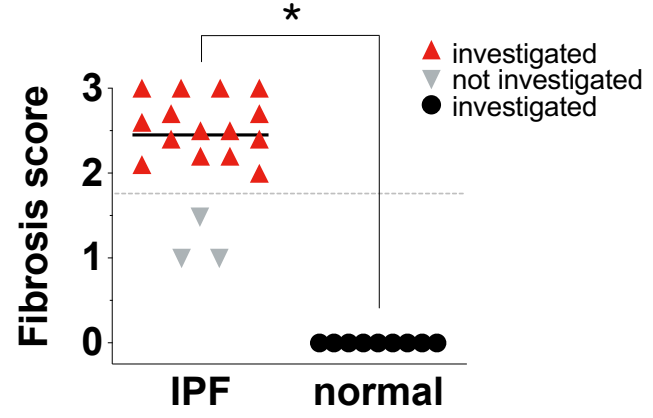

Supplementary Figure 1 | Characterization of human IPF and normal lung tissues. (A) Donor characteristics of IPF and normal lung tissues. (B) Representative chest radiographs of IPF and normal lung donors. Arrows indicate changed lung shape, decreased lung volume, and increased radiopacity consistent with pulmonary fibrosis. (C) Fibrosis scoring rubric used to assess the extent of architectural disruption and fibrosis in human lung tissues. (D) Overview of the description and quantity of tissue samples analyzed in this study. (E) Samples were collected from three regions (i.e., medial, lateral, peripheral) of right middle lobes. (F) Demonstration of quantitative image analysis method used for fibrosis scoring. For each fibrosis score, a weighted average is calculated from the ratios of the total area. Five high-power fields were analyzed per region, and an average fibrosis score was calculated for each region. (G) Fibrosis scores of all regions of IPF and normal lungs investigated in this study. Only regions of IPF lungs with fibrosis score $\geq 2$ (red triangle) were investigated in this study. ${ }^{*} p<0.001$. 


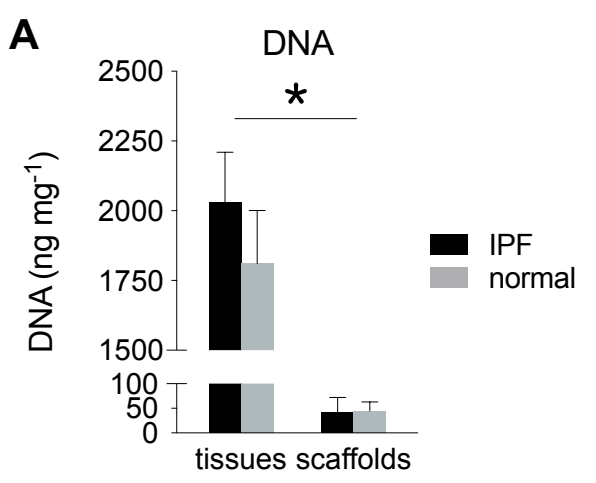

B

\begin{tabular}{lrr} 
Scaffolds & Sterility & Mycoplasma \\
\hline IPF lung 1 & NEGATIVE & NEGATIVE \\
IPF lung 2 & NEGATIVE & NEGATIVE \\
IPF lung 3 & NEGATIVE & NEGATIVE \\
Normal lung 1 & NEGATIVE & NEGATIVE \\
Normal lung 2 & NEGATIVE & NEGATIVE \\
Normal lung 3 & NEGATIVE & NEGATIVE
\end{tabular}

Supplementary Figure 2 | Quality control assays of IPF and normal lung matrix scaffolds. Quantification of (A) DNA to confirm removal of nuclear material from IPF and normal lung matrix scaffolds. (B) Results of sterility and mycoplasma assays. Prior to use in studies, IPF and normal lung matrix scaffolds were tested for absence of bacteria and fungi. Scaffolds were also tested for absence of mycoplasma using MycoAlert PLUS Mycoplasma Detection Assay. ${ }^{*} p<0.001$. 


\begin{tabular}{lllll} 
Antibody & Application & Vendor & Product number & Dilution \\
\hline Rabbit anti-alpha smooth muscle actin & IHC-P & Cell Signaling Technology & 19245 & $1: 500$ \\
Rabbit anti-fibrillin 2 & IHC-P & Sigma Life Science & HPA012853 & $1: 50$ \\
Rabbit anti-Ki67 & IHC-P & ThermoFisher Scientific & PA1-38032 & $1: 500$ \\
Rabbit anti-laminin Y1 & IHC-P & Abcam & ab233389 & $1: 2000$ \\
Rabbit anti-matrix gla protein & IHC-P & LS Bio & LS-B14824 & $1: 100$ \\
Rabbit anti-periostin & IHC-P & Abcam & ab14041 & $1: 300$
\end{tabular}

\begin{tabular}{llll} 
ELISA kit & Application & Vendor & Product number \\
\hline Human basic fibroblast growth factor & Cell culture media & R\&D Systems & DFB50 \\
Human transforming growth factor $\beta$ & Cell culture media & R\&D Systems & DB100B
\end{tabular}

Supplementary Table 1 | Antibodies and ELISA kits. 


\begin{tabular}{lll} 
Gene & Forward primer & Reverse primer \\
\hline GAPDH & TGCACCACCAACTGCTTAGC & GGCATGGACTGTGGTCATGAG \\
MMP2 & CTTCCAAGTCTGGAGCGATGT & TACCGTCAAAGGGGTATCCAT \\
COL1A1 & TCTGCGACAACGGCAAGGTG & GACGCCGGTGGTTTCTTGGT \\
LAMC1 & TGGGCATTCTTCTGTCTGTACAA & GCCACCCATCCTCATCAATC \\
ACTA2 & CCTGGCTTCGCTGTCTACCT & TTGCGGTGGACGATGGA \\
PDGFC & GGACTCAGGCGGAATCCAA & CTGAGGATCTTGTACTCCGTTCTG \\
YAP1 & ACGTTCATCTGGGACAGCAT & GTTGGGAGATGGCAAAGACA \\
PRRX1 & CAGATTGGTGGCTGTTAGATTGAA & GATGCACTTTTAGCACACATTTGTATT \\
PTEN & CAAGATGATGTTTGAAACTATTCCAATG & CCTTTAGCTGGCAGACCACAA
\end{tabular}

\section{Supplementary Table 2 | Primers.}


IPF lung donor demographics and clinical characteristics

\begin{tabular}{llll}
\hline Characteristics & Donor 1 & Donor 2 & Donor 3 \\
\hline Sex & Female & Male & Female \\
Age (years) & 62 & 66 & 55 \\
Height $(\mathrm{cm})$ & 163 & 163 & 155 \\
Weight $(\mathrm{kg})$ & 59 & 64 & 42 \\
BMI $\left(\mathrm{kg} \mathrm{m}^{-2}\right)$ & 22 & 24 & 18 \\
Smoking history (pack years) & 0 & 0 & 0 \\
Lung disease & IPF & IPF & IPF \\
Lung allocation score & 36.1 & 83.5 & 45.5 \\
Radiologic characteristics & UIP, reticular opacities & UIP, honeycombing & UIP, reticular opacities
\end{tabular}

NORMAL lung donor demographics and clinical characteristics

\begin{tabular}{llll}
\hline Characteristics & Donor 1 & Donor 2 & Donor 3 \\
\hline Sex & Female & Male & Male \\
Age (years) & 24 & 56 & 52 \\
Height $(\mathrm{cm})$ & 165 & 175 & 178 \\
Weight $(\mathrm{kg})$ & 81 & 63 & 99 \\
BMI $\left(\mathrm{kg} \mathrm{m}^{-2}\right)$ & 30 & 20 & 31 \\
Smoking history (pack years) & 0 & 0 & 0 \\
Lung disease & None & None & None \\
Lung allocation score & 0 & 0 & 0 \\
Radiologic characteristics & Abnormal basilar aeration & Cardiac enlargement & Trace pleural effusion \\
\end{tabular}

Supplementary Table 3 | Demographics and clinical characteristics of human lung donors. UIP, usual interstitial pneumonia. 


\begin{tabular}{|c|c|c|c|c|}
\hline \multirow[b]{2}{*}{ Growth factor } & \multirow[b]{2}{*}{ Description } & \multicolumn{2}{|c|}{ Concentration $\left(\mathrm{pg} \mathrm{mL}^{-1}\right)$} & \multirow{2}{*}{$\begin{array}{l}\text { Fold change } \\
\text { from normal }\end{array}$} \\
\hline & & Normal tissue & IPF tissue & \\
\hline TGF- $\beta 3$ & Transforming growth factor $\beta 3$ & ND & 65.8 & * \\
\hline HB-EGF & Heparin-binding EGF-like growth factor & ND & 4.2 & * \\
\hline IGFBP-1 & Insulin-like growth factor binding protein 1 & 0.5 & 86.8 & $159.5 \Delta$ \\
\hline bFGF & Basic fibroblast growth factor & 10.1 & 213.8 & $21.2 \Delta$ \\
\hline EG-VEGF & Endocrine gland-derived vascular endothelial growth factor & 2.2 & 38.1 & $17.3 \Delta$ \\
\hline BDNF & Brain-derived neurotrophic factor & 31.1 & 145.4 & $4.7 \Delta$ \\
\hline GDF-15 & Growth differentiation factor 15 & 97.8 & 243.4 & $2.5 \mathrm{~A}$ \\
\hline PDGF-AA & Platelet-derived growth factor $\mathrm{AA}$ & 228.1 & 486.7 & $2.1 \mathrm{~A}$ \\
\hline IGFBP-6 & Insulin-like growth factor binding protein 6 & 123.0 & 228.5 & $1.9 \boldsymbol{A}$ \\
\hline HGF & Hepatocyte growth factor & 13313.2 & 19831.9 & $1.5 \Delta$ \\
\hline VEGF & Vascular endothelial growth factor & 135.9 & 158.8 & $1.2 \Delta$ \\
\hline EGF R & Epidermal growth factor receptor & 17343.2 & 12828.8 & $0.7 \nabla$ \\
\hline OPG & Osteoprotegerin & 60.8 & 33.8 & $0.6 \nabla$ \\
\hline
\end{tabular}

Supplementary Table 4 | Quantification of growth factors in IPF and normal lung tissues. Growth factor concentrations were measured by multiplex growth factor array. Green arrow $(\Delta)$ indicates positive fold change (increase) from normal in concentration of growth factors. Red arrow $(\boldsymbol{\nabla})$ indicates negative fold change (decrease) from normal in concentration of growth factors. ND: not detected. ${ }^{*}$ IPF-specific growth factor not detected in normal lung tissue. 